\title{
Regional gravity modeling in terms of spherical base functions
}

\author{
Michael Schmidt • Martin Fengler • \\ Torsten Mayer-Gürr • Annette Eicker • \\ Jürgen Kusche • Laura Sánchez • Shin-Chan Han
}

Received: 25 August 2006 / Accepted: 25 August 2006 / Published online: 17 October 2006

(C) Springer-Verlag 2006

\begin{abstract}
This article provides a survey on modern methods of regional gravity field modeling on the sphere. Starting with the classical theory of spherical harmonics, we outline the transition towards space-localizing methods such as spherical splines and wavelets. Special emphasis is given to the relations among these methods, which all involve radial base functions. Moreover, we provide extensive applications of these methods and numerical results from real space-borne data of
\end{abstract}

M. Schmidt $(\varangle) \cdot$ L. Sánchez

Deutsches Geodätisches Forschungsinstitut (DGFI),

Alfons-Goppel-Strasse 11, 80539 Munich, Germany

e-mail: schmidt@dgfi.badw.de

L. Sánchez

e-mail: sanchez@dgfi.badw.de

M. Fengler

Geomathematics Group,

Technical University of Kaiserslautern, P.O. Box 3049,

67653 Kaiserslautern, Germany

e-mail: fengler@mathematik.uni-kl.de

T. Mayer-Gürr · A. Eicker

Institute of Theoretical Geodesy,

University of Bonn, Nussallee 17, 53115 Bonn, Germany

e-mail: mayer-guerr@geod.uni-bonn.de

A. Eicker

e-mail: eicker@geod.uni-bonn.de

J. Kusche

Delft Institute of Earth Observation and Space Systems

(DEOS), Delft University of Technology, Kluyverweg 1,

P.O. Box 5058, 2600 GB Delft, The Netherlands

e-mail: j.kusche@1r.tudelft.nl

S.-C. Han

Geodetic Science, Department of Geological Sciences,

Ohio State University, Columbus, USA

e-mail: han.104@osu.edu recent satellite gravity missions, namely the Challenging Minisatellite Payload (CHAMP) and the Gravity Recovery and Climate Experiment (GRACE). We also derive high-resolution gravity field models by effectively combining space-borne and surface measurements using a new weighted level-combination concept. In addition, we outline and apply a strategy for constructing spatiotemporal fields from regional data sets spanning different observation periods.

Keywords Regional gravity modeling - Spherical radial base functions - Multi-resolution representation . Spherical wavelets . Challenging Minisatellite Payload (CHAMP) and Gravity Recovery and Climate Experiment (GRACE)

\section{Introduction}

At the time when the new global Earth Gravity Model EGM06, i.e., a spherical harmonic expansion of the geopotential up to degree and order 2160 (Pavlis et al.2005), will become available, it makes sense to highlight some appropriate approaches for regional gravity modeling. Although technically the analysis and synthesis may be possible for ultra-high expansions when using tailored stable algorithms for the evaluation of the associated Legendre functions, it is well-known that spherical harmonic models cannot represent terrestrial data of heterogeneous density and quality in a proper way.

On the other hand, gravity field modeling in terms of spherical (radial) base functions has long been considered as an alternative to this classical procedure. Regional gravity models have been routinely developed 
based on terrestrial (surface) data using either regular predefined systems of spherical base functions, like those generated by point masses and higher-order multipoles (e.g., Cui 1995), or data-driven irregular systems as within the least-squares collocation (LSC) technique (e.g., Sansò and Tscherning 2003) or with sequential multipole methods (e.g., Marchenko 1998). Regarding data-driven irregular point systems, Mautz et al. (2004) estimate the positions of locally supported base functions using global optimization methods.

Discrete approximation methods with spherical, radial-symmetric harmonic base functions arise naturally from the discretization of integral operators (e.g. the Stokes operator) that relate geodetic observables to the disturbing potential or the surface-layer density, if we restrict ourselves to the sphere. However, there are more general ways to introduce an operable concept of spherical base functions through discretizing spherical convolution integrals. This idea follows Freeden and Schreiner (1995) and Freeden and Windheuser (1996) and opens the door to space-discrete spherical wavelet approximations of the gravity field and the implementation of multi-resolution techniques in spherical base function modeling. Thus, this work is largely based on the results of Freeden et al. (1998a), Freeden (1999) as well as Freeden and Michel (2004).

The application of such techniques to real spaceborne and surface gravity data came only recently into fruition, e.g., Fengler et al. (2004a,b) and Schmidt et al. (2005a,b, 2006). Besides gravity field modeling, the application of scalar, vector and tensor spherical wavelets has become more and more popular in other fields of geodesy and geophysics, e.g., the analysis of measurements of the surface air temperature (Li 1999), the Earth's magnetic field (Holschneider et al. 2003, Maier 2005, Panet et al. 2005), the modeling of ionospheric currents (Mayer 2004), atmospheric flows (Fengler 2005) and oceanographic flows (Freeden et al. 2005).

The objective of this article is to provide a consistent overview about several modern methods of regional gravity field modeling. To achieve this, we present an extensive study in theory and application of the evaluation of real gravity data from the Challenging Minisatellite Payload (CHAMP) and Gravity Recovery and Climate Experiment (GRACE) satellite missions in order to constitute regional gravity models using series expansions in spherical splines and wavelets. In addition, we construct a regional high-resolution gravity model from satellite and surface data applying a specific combination concept and introduce an appropriate strategy for implementing the time-dependency into the representation.
We discuss and apply numerical integration techniques and parameter estimation methods, considering both space-borne and surface data. Regarding the determination of model parameters from estimation methods, the potentially large number of base functions and the size of the resulting linear equation systems may hamper application in practice, but in this way one cannot only provide statistical information for the estimated parameters, but also apply data-driven concepts in regularization, data combination and coefficient thresholding. In view of discretizing a spherical convolution integral, we place special emphasis on the choice of the point systems, the choice of the base functions, as well as practical questions like data combination.

This work is organized as follows: In the Sect. 2, we present fundamental concepts involving spherical harmonics and spherical base functions. We largely follow the notation of Freeden et al. (1998a), and outline the relationship to spherical convolutions. Modeling concepts based on spherical splines and wavelets are treated, as well as the multi-resolution representation of a given input signal. The third section deals with two evaluation methods in order to determine the coefficients of the spherical base function representation. Starting with numerical integration techniques, we point out the link to appropriate parameter estimation procedures. In order to demonstrate the power of spherical base function modeling, we present in the Sect. 4 different regional representations of the gravity field for the northern part of South America computed from real CHAMP and GRACE data as well as from surface data.

\section{Spherical modelling}

\subsection{Spherical harmonics}

Let $\boldsymbol{x}=\left(x_{1}, x_{2}, x_{3}\right)^{\mathrm{T}}$ and $\boldsymbol{y}=\left(y_{1}, y_{2}, y_{3}\right)^{\mathrm{T}}$ be vectors of the three-dimensional Euclidean space $\mathbb{R}^{3}$. Then $\boldsymbol{x}^{\mathrm{T}} \boldsymbol{y}=$ $\sum_{i=1}^{3} x_{i} y_{i}$ is referred to as the inner product. The corresponding norm is given by $|\boldsymbol{x}|=\sqrt{\boldsymbol{x}^{\mathrm{T}} \boldsymbol{x}}$. Any vector $\boldsymbol{x} \in \mathbb{R}^{3} \backslash\{\mathbf{0}\}$ is uniquely represented as $\boldsymbol{x}=r \boldsymbol{\xi}$, where $r=|\boldsymbol{x}|$ and $|\boldsymbol{\xi}|=1$. Furthermore, let $\Omega_{R}^{\text {int }}$ and $\Omega_{R}^{\text {ext }}$ denote the inner and outer space of the sphere $\Omega_{R}$ with radius $R ; \overline{\Omega_{R}^{\mathrm{ext}}}$ is defined as $\overline{\Omega_{R}^{\mathrm{ext}}}=\Omega_{R}^{\mathrm{ext}} \cup \Omega_{R}$ and $\Omega_{1}=: \Omega$ means the unit sphere.

As customary, the space of all real square-integrable functions $F$ on $\Omega_{R}$ is called $L^{2}\left(\Omega_{R}\right) . L^{2}\left(\Omega_{R}\right)$ is a Hilbert space with the inner product

$$
\langle F, G\rangle_{L^{2}\left(\Omega_{R}\right)}=\int_{\Omega_{R}} F(\boldsymbol{x}) G(\boldsymbol{x}) \mathrm{d} \Omega_{R}(\boldsymbol{x})
$$


for $F, G \in L^{2}\left(\Omega_{R}\right)$ and the associated norm $\|F\|_{L^{2}\left(\Omega_{R}\right)}=$ $\sqrt{\langle F, F\rangle_{L^{2}\left(\Omega_{R}\right)}} ; \mathrm{d} \Omega_{R}(\boldsymbol{x})$ denotes the surface element on the sphere $\Omega_{R}$.

The real-valued (surface) spherical harmonics $Y_{n, m}(\xi)$ of degree $n$ and order $m$ form a complete orthonormal basis of $L^{2}(\Omega)$, e.g., Heiskanen and Moritz (1967). Hence, each function (signal) $F \in L^{2}(\Omega)$ can be uniquely written in $L^{2}(\Omega)$-sense as Fourier series

$F(\xi)=\sum_{n=0}^{\infty} \sum_{m=-n}^{n} F_{n, m} Y_{n, m}(\xi)$

with $\xi \in \Omega$. The Stokes coefficients $F_{n, m}$ are computable via the spherical Fourier transform $F_{n, m}=\langle F$, $\left.Y_{n, m}\right\rangle_{L^{2}(\Omega)}$. As another important ingredient, we require the Legendre polynomials $P_{n}(t)$ of degree $n$ which are, e.g., obtainable via the Rodriguez formula

$P_{n}(t)=\frac{1}{2^{n} n !} \frac{\mathrm{d}^{n}}{\mathrm{~d} t^{n}}\left(t^{2}-1\right)^{n}, \quad t \in[-1,1]$.

Altogether, we end up at the spherical addition theorem

$\sum_{m=-n}^{n} Y_{n, m}(\xi) Y_{n, m}(\boldsymbol{\eta})=\frac{2 n+1}{4 \pi} P_{n}\left(\xi^{\mathrm{T}} \boldsymbol{\eta}\right)$

with $\xi, \eta \in \Omega$ connecting the spherical harmonics and the Legendre polynomials (Freeden et al. 1998a). Equation (4) forms the foundation in formulating scaling functions and wavelets on the sphere; see also the comments in the context of Eq. (19). Moreover, we gather the $2 n+1$ spherical harmonics $Y_{n, m}(\xi)$ of degree $n$ and order $m=-n, \ldots, n$ into the finite-dimensional Hilbert space $H_{n}(\Omega)$, and consequently, all spherical harmonics $Y_{n, m}(\boldsymbol{\xi})$ of degree $n=0, \ldots, n^{\prime}$ and order $m=-n, \ldots, n$ into the Hilbert space $H_{0, \ldots, n^{\prime}}(\Omega)$ of dimension

$\operatorname{dim}\left(H_{0, \ldots, n^{\prime}}(\Omega)\right)=\left(n^{\prime}+1\right)^{2}=: \bar{n}$.

In the sequel, we mostly identify $F$ with the gravitational potential or the disturbing gravitational potential of the Earth. Given $F$ on the sphere $\Omega_{R}$, i.e., $F \in L^{2}\left(\Omega_{R}\right)$, we can write the upward continuation by

$F(\boldsymbol{x})=\sum_{n=0}^{\infty} \sum_{m=-n}^{n} F_{n, m} H_{n, m}^{R}(\boldsymbol{x})$

with $\boldsymbol{x}=r \boldsymbol{\xi} \in \overline{\Omega_{R}^{\mathrm{ext}}}$ and $F_{n, m}=\left\langle F, H_{n, m}^{R}\right\rangle_{L^{2}\left(\Omega_{R}\right)}$. The functions

$H_{n, m}^{R}(\boldsymbol{x})=\frac{1}{R}\left(\frac{R}{r}\right)^{n+1} Y_{n, m}(\boldsymbol{\xi})$ are known as outer or solid spherical harmonics. Consequently, we define the space $H_{n}\left(\overline{\Omega_{R}^{\mathrm{ext}}}\right)$ of all linear combinations of the $2 n+1$ outer spherical harmonics $H_{n, m}^{R}(\boldsymbol{x})$ of degree $n$ and order $m=-n, \ldots, n$ as well as the space $H_{0, \ldots, n^{\prime}}\left(\overline{\Omega_{R}^{\text {ext }}}\right)$ of all outer spherical harmonics $H_{n, m}^{R}(\boldsymbol{x})$ of degree $n=0, \ldots, n^{\prime}$ and order $m=-n, \ldots, n$. Finally, we mention that the $L^{2}\left(\Omega_{R}\right)$ norm of a signal $F \in L^{2}\left(\Omega_{R}\right)$ can be interpreted as the energy content or the global root-mean-square (RMS) value of $F$. By a degree-wise decomposition of the $L^{2}\left(\Omega_{R}\right)$-norm, we obtain

$\|F\|_{L^{2}\left(\Omega_{R}\right)}^{2}=\sum_{n=0}^{\infty} \sigma_{n}^{2}(F)$,

where $\sigma_{n}^{2}(F)=\sum_{m=-n}^{n} F_{n, m}^{2}$ are the well-known degree variances of $F$, e.g., Heiskanen and Moritz (1967) or Torge (2001). If we assume that the signal $F(\boldsymbol{x})$ is bandlimited, i.e. $F \in H_{0, \ldots, n^{\prime}}\left(\overline{\Omega_{R}^{\mathrm{ext}}}\right)$, we can rewrite Eq. (6) as

$F(\boldsymbol{x})=\boldsymbol{h}(\boldsymbol{x})^{\mathrm{T}} \boldsymbol{f}^{\wedge}$

with $\boldsymbol{x} \in \overline{\Omega_{R}^{\mathrm{ext}}}$. Herein $\boldsymbol{f}^{\wedge}$ and $\boldsymbol{h}(\boldsymbol{x})$ denote $\bar{n} \times 1$ vectors given by

$$
\begin{aligned}
\boldsymbol{f}^{\wedge} & =\left(F_{0,0}, F_{1,-1}, \ldots, F_{n^{\prime}, n^{\prime}}\right)^{\mathrm{T}}, \\
\boldsymbol{h}(\boldsymbol{x}) & =\left(H_{0,0}^{R}(\boldsymbol{x}), H_{1,-1}^{R}(\boldsymbol{x}), \ldots, H_{n^{\prime}, n^{\prime}}^{R}(\boldsymbol{x})\right)^{\mathrm{T}} .
\end{aligned}
$$

Unless otherwise noted in the following, we always assume that $F(\boldsymbol{x})$ is band-limited, i.e., $F \in H_{0, \ldots, n^{\prime}}\left(\overline{\Omega_{R}^{\mathrm{ext}}}\right)$.

\subsection{Spherical base functions}

Writing Eq. (9) for altogether $N$ position vectors $\boldsymbol{x}=\boldsymbol{x}_{k}$ with $k=1, \ldots, N$ and $\boldsymbol{x}_{k} \in \Omega_{R}$, the linear equation system

$\boldsymbol{f}=\boldsymbol{H} \boldsymbol{f}^{\wedge}$

results, wherein

$\boldsymbol{f}=\left(F\left(\boldsymbol{x}_{1}\right), F\left(\boldsymbol{x}_{2}\right), \ldots, F\left(\boldsymbol{x}_{N}\right)\right)^{\mathrm{T}}$

is the $N \times 1$ vector of the signal values $F\left(\boldsymbol{x}_{k}\right)$, and

$\boldsymbol{H}=\left(\boldsymbol{h}\left(\boldsymbol{x}_{1}\right), \boldsymbol{h}\left(\boldsymbol{x}_{2}\right), \ldots, \boldsymbol{h}\left(\boldsymbol{x}_{N}\right)\right)^{\mathrm{T}}$

is an $N \times \bar{n}$ matrix. If $N$ exceeds the number $\bar{n}$ of unknown Stokes coefficients, Eq. (12) can be solved via

$\boldsymbol{f}^{\wedge}=\left(\boldsymbol{H}^{\mathrm{T}} \boldsymbol{H}\right)^{-1} \boldsymbol{H}^{\mathrm{T}} \boldsymbol{f}$, 
as long as the matrix $\boldsymbol{H}$ possesses full column rank; see e.g., Koch (1999). In this case, the system

$S_{N}=\left\{\boldsymbol{x}_{k} \in \Omega_{R} \mid k=1, \ldots, N\right\}$

of points $\boldsymbol{x}_{k}$ is called admissible. Even if the equality $N=\bar{n}$ holds, the matrix $\boldsymbol{H}$ is regular and $S_{N}$ is called fundamental (Freeden et al. 1998a). In the following, we always assume that the point system $S_{N}$ is at least admissible.

Substituting Eq. (15) into Eq. (9) yields the interpolation formula $F(\boldsymbol{x})=\boldsymbol{h}(\boldsymbol{x})^{\mathrm{T}}\left(\boldsymbol{H}^{\mathrm{T}} \boldsymbol{H}\right)^{-1} \boldsymbol{H}^{\mathrm{T}} \boldsymbol{f}$, which can be rewritten as

$F(\boldsymbol{x})=\sum_{k=1}^{N} F\left(\boldsymbol{x}_{k}\right) Z\left(\boldsymbol{x}, \boldsymbol{x}_{k}\right)$

The functions $Z\left(\boldsymbol{x}, \boldsymbol{x}_{k}\right)=\boldsymbol{h}\left(\boldsymbol{x}_{k}\right)^{\mathrm{T}}\left(\boldsymbol{H}^{\mathrm{T}} \boldsymbol{H}\right)^{-1} \boldsymbol{h}(\boldsymbol{x})$ depend not only on $\boldsymbol{x}$ and $\boldsymbol{x}_{k}$, but also - due to the matrix $\boldsymbol{H}$ - on all points of the admissible system $S_{N}$. One may argue that, in addition, we cannot expect a decay of $Z\left(\boldsymbol{x}, \boldsymbol{x}_{k}\right)$ as the computation point $\boldsymbol{x}$ moves away from the data point $\boldsymbol{x}_{k}$. However, for regional or local representations, we would prefer a "two-point" function $B\left(\boldsymbol{x}, \boldsymbol{x}_{k}\right)$ that allows the computation of $F(\boldsymbol{x})$ mainly just from signal values given in the vicinity of $\boldsymbol{x}$, i.e., which is characterized by the ability to localize. For this purpose, we introduce the representation

$F(\boldsymbol{x})=\sum_{k=1}^{N} c_{k} B\left(\boldsymbol{x}, \boldsymbol{x}_{k}\right)$

of the band-limited function $F$ in terms of spherical base functions $B\left(\boldsymbol{x}, \boldsymbol{x}_{k}\right), k=1, \ldots, N$ defined by the Legendre series

$B\left(\boldsymbol{x}, \boldsymbol{x}_{k}\right)=\sum_{n=0}^{n^{\prime}} \frac{2 n+1}{4 \pi R^{2}}\left(\frac{R}{r}\right)^{n+1} B_{n} P_{n}\left(\xi^{\mathrm{T}} \xi_{k}\right)$

with $\boldsymbol{x}=r \boldsymbol{\xi} \in \overline{\Omega_{R}^{\mathrm{ext}}}$ and $\boldsymbol{x}_{k}=R \boldsymbol{\xi}_{k} \in \Omega_{R}$.

The initially unknown coefficients $c_{k}$ in Eq. (18) play a similar role as the Stokes coefficients $F_{n, m}$ of the spherical harmonic approach. For $\boldsymbol{x} \in \Omega_{R}$, i.e. $r=R$, the spherical base function $B\left(\boldsymbol{x}, \boldsymbol{x}_{k}\right)$ depends only on the spherical distance $\alpha=\arccos \left(\boldsymbol{\xi}^{\mathrm{T}} \boldsymbol{\xi}_{k}\right)$. Thus, $B\left(\boldsymbol{x}, \boldsymbol{x}_{k}\right)$ is rotationally symmetric and the spherical analogue to radial base functions (Narcowich and Ward 1996). In the sequel, we always will refer to spherical base functions, but having radial base functions in mind.

Since the Legendre coefficients $B_{n}$ in Eq. (19) reflect the spectral behavior, the total $L^{2}\left(\Omega_{R}\right)$-norm of $B\left(\boldsymbol{x}, \boldsymbol{x}_{k}\right)$ is computable according to Eq. (8) and reads

$\|B\|_{L^{2}\left(\Omega_{R}\right)}^{2}=\sum_{n=0}^{n^{\prime}} \sigma_{n}^{2}(B)$,

wherein the degree variances

$\sigma_{n}^{2}(B)=\frac{2 n+1}{4 \pi R^{2}} B_{n}^{2}$

constitute the power spectrum of $B\left(\boldsymbol{x}, \boldsymbol{x}_{k}\right)$. For an alternative approach involving wavelet variances, see Freeden and Michel (2004) and Fengler et al. (2006b).

With the two $N \times 1$ vectors

$$
\begin{aligned}
\boldsymbol{c} & =\left(c_{1}, c_{2}, \ldots, c_{N}\right)^{\mathrm{T}}, \\
\boldsymbol{b}(\boldsymbol{x}) & =\left(B\left(\boldsymbol{x}, \boldsymbol{x}_{1}\right), B\left(\boldsymbol{x}, \boldsymbol{x}_{2}\right), \ldots, B\left(\boldsymbol{x}, \boldsymbol{x}_{N}\right)\right)^{\mathrm{T}}
\end{aligned}
$$

of coefficients $c_{k}$ and spherical base functions $B\left(\boldsymbol{x}, \boldsymbol{x}_{k}\right)$, Eq. (18) reads

$F(\boldsymbol{x})=\boldsymbol{b}(\boldsymbol{x})^{\mathrm{T}} \boldsymbol{c}$.

In order to guarantee that Eq. (24) equals Eq. (9) we require

$H_{0, \ldots, n^{\prime}}\left(\overline{\Omega_{R}^{\mathrm{ext}}}\right)=\operatorname{span}\left\{B\left(\boldsymbol{x}, \boldsymbol{x}_{k}\right) \mid k=1, \ldots, N\right\}$.

To clarify this statement, we introduce the $\bar{n} \times \bar{n}$ diagonal matrix $\boldsymbol{B}=\operatorname{diag}\left(B_{0}, B_{1}, B_{1}, B_{1}, B_{2}, \ldots, B_{n^{\prime}}\right)$ and consider the addition theorem of spherical harmonics (Eq. 4) in Eq. (19). With $B\left(\boldsymbol{x}, \boldsymbol{x}_{k}\right)=\boldsymbol{h}\left(\boldsymbol{x}_{k}\right)^{\mathrm{T}} \boldsymbol{B} \boldsymbol{h}(\boldsymbol{x})$ the transformation

$\boldsymbol{b}(\boldsymbol{x})=\boldsymbol{H} \boldsymbol{B} \boldsymbol{h}(\boldsymbol{x})$

states that Eq. (25) is fulfilled if both the point system $S_{N}$ is admissible and the matrix $\boldsymbol{B}$ is positive definite, i.e. the Legendre coefficients $B_{n}$ are restricted to the condition (Schmidt et al. 2005a)

$B_{n}>0$ for $n=0, \ldots, n^{\prime}$.

Since usually the number $N$ of admissible points exceeds the number $\bar{n}$ (Eq. 5), only $\bar{n}$ functions $B\left(\boldsymbol{x}, \boldsymbol{x}_{k}\right)$ are linearly independent. Substituting Eq. (26) into Eq. (24) and comparing the result with Eq. (9) yields the relation

$\boldsymbol{f}^{\wedge}=\boldsymbol{B} \boldsymbol{H}^{\mathrm{T}} \boldsymbol{c}$.

Hence, the Stokes coefficients $F_{n, m}$ with $n=0, \ldots, n^{\prime}$ and $m=-n, \ldots, n$ can be determined by means of the coefficient vector $\boldsymbol{c}$. 
The comparison of the vectors $\boldsymbol{h}(\boldsymbol{x})$ and $\boldsymbol{b}(\boldsymbol{x})$, defined in Eqs. (11) and (23), exposes the difference between the representations (Eqs. 9 and 24) of the function $F(\boldsymbol{x})$ in terms of spherical harmonics and spherical base functions, respectively. Whereas the elements of $\boldsymbol{h}(\boldsymbol{x})$, i.e., the outer harmonics $H_{n, m}^{R}(\boldsymbol{x})$, depend on the degree $n$ and order $m$, the elements $B\left(\boldsymbol{x}, \boldsymbol{x}_{k}\right)$ of $\boldsymbol{b}(\boldsymbol{x})$ depend on the spatial position vectors $\boldsymbol{x}_{k}$. Consequently, regional or local structures of a function (input signal) $F(\boldsymbol{x})$ are better described by Eq. (18) in terms of spherical base functions (e.g., Freeden and Michel 2004).

The choice of the spherical base functions depends mainly on the application. A well-established strategy is to construct spherical base functions $B\left(\boldsymbol{x}, \boldsymbol{x}_{k}\right)$ in a way that their power spectrum corresponds to the power spectrum of the signal $F(\boldsymbol{x})$, which shall be modeled according to Eq. (18). To be more specific, we start with the energy representations (Eqs. 8 and 20) for the input signal and the spherical base function, set $\sigma_{n}^{2}(F)=\sigma_{n}^{2}(B)$ for $n=0, \ldots, n^{\prime}$ and obtain

$$
B_{n}=\sqrt{\frac{4 \pi R^{2}}{2 n+1}} \sigma_{n}(F)
$$

With this choice of the Legendre coefficients $B_{n}$, the function $B\left(\boldsymbol{x}, \boldsymbol{x}_{k}\right)$ (Eq. 19) corresponds to the covariance function used in LSC (e.g., Moritz 1980) and may be considered as a harmonic spline function (e.g., Freeden 1981). If, instead, the Riesz representers of the observation functionals are used as base functions (which are radial-symmetric for isotropic observation functionals), our approach for representing the potential becomes identical to LSC (Moritz 1980).

Many other examples of spherical base functions are listed in Freeden et al. (1998a) and in the references within this textbook. Here, we refer to the end of Sect. 2.4 of this paper.

\subsection{Spherical convolution}

Equation (18) can also be embedded into the much more general concept of spherical convolution. A spherical convolution means the basic tool for low- and bandpass filtering processes. In Sect. 2.4, we will apply it in order to constitute a multi-resolution representation of the input signal $F(\boldsymbol{x})$.

To study the spherical convolution in more detail, we first introduce the unique reproducing kernel

$$
K_{\mathrm{rep}}\left(\boldsymbol{x}, \boldsymbol{x}_{k}\right)=\sum_{n=0}^{n^{\prime}} \frac{2 n+1}{4 \pi R^{2}}\left(\frac{R}{r}\right)^{n+1} P_{n}\left(\xi^{\mathrm{T}} \xi_{k}\right)
$$

of the space $H_{0, \ldots, n^{\prime}}\left(\overline{\Omega_{R}^{\text {ext }}}\right)$ fulfilling the condition [e.g. Moritz (1980) or Freeden (1999)]

$F(\boldsymbol{x})=\left(K_{\mathrm{rep}} * F\right)(\boldsymbol{x})$.

In Eq. (31) the spherical convolution $K_{\text {rep }} * F$ is defined as

$\left(K_{\text {rep }} * F\right)(\boldsymbol{x})=\left\langle F, K_{\mathrm{rep}}(\cdot, \boldsymbol{x})\right\rangle_{L^{2}\left(\Omega_{R}\right)}$.

The substitution of Eq. (31) into Eqs. (18) and (24) yields

$\left(K_{\mathrm{rep}} * F\right)(\boldsymbol{x})=\sum_{k=1}^{N} c_{k} B\left(\boldsymbol{x}, \boldsymbol{x}_{k}\right)=\boldsymbol{b}(\boldsymbol{x})^{\mathrm{T}} \boldsymbol{c}$.

Since $F(\boldsymbol{x})$ is an element of $H_{0, \ldots, n^{\prime}}\left(\overline{\Omega_{R}^{\text {ext }}}\right)$, we rewrite the convolution $K_{\mathrm{rep}} * F$ from the right-hand side of Eq. (31) as a series expansion in spherical base functions $K_{\text {rep }}\left(\boldsymbol{x}, \boldsymbol{x}_{k}\right)$, i.e.,

$\left(K_{\mathrm{rep}} * F\right)(\boldsymbol{x})=\sum_{k=1}^{N} d_{k} K_{\mathrm{rep}}\left(\boldsymbol{x}, \boldsymbol{x}_{k}\right)=\boldsymbol{k}_{\mathrm{rep}}(\boldsymbol{x})^{\mathrm{T}} \boldsymbol{d}$.

The $N \times 1$ vectors $\boldsymbol{d}$ and $\boldsymbol{k}_{\text {rep }}(\boldsymbol{x})$ are defined analogously to Eqs. (22) and (23). This result states that the original representation (Eq. 18) of $F(\boldsymbol{x})$ in spherical base functions $B\left(\boldsymbol{x}, \boldsymbol{x}_{k}\right)$ can be replaced by Eq. (34) in terms of the reproducing kernel (Eq. 30). Note, that we assume the same admissible point system $S_{N}$ (Eq. 16) in Eqs. (33) and (34).

Before discussing the relation between the two coefficient vectors $\boldsymbol{c}$ and $\boldsymbol{d}$ in more detail, we require a result that is of great importance for low- and band-pass filtering processes.

Theorem 1 Let $\bar{F} \in L^{2}\left(\Omega_{R}\right)$ and

$K\left(\boldsymbol{x}, \boldsymbol{x}_{k}\right)=\sum_{n=0}^{\infty} \frac{2 n+1}{4 \pi R^{2}}\left(\frac{R}{r}\right)^{n+1} K_{n} P_{n}\left(\boldsymbol{\xi}^{\mathrm{T}} \boldsymbol{\xi}_{k}\right)$

be a kernel with

$K_{n}\left\{\begin{array}{lll}\neq 0 & \text { for } & n=0, \ldots, n^{\prime} \\ =0 & \text { for } & n>n^{\prime}\end{array}\right.$.

Assume further that for $\boldsymbol{x}=r \xi \in \overline{\Omega_{R}^{\mathrm{ext}}}$ and $\boldsymbol{x}_{k}=R \xi_{k} \in$ $S_{N} \subset \Omega_{R}$

$(K * \bar{F})(\boldsymbol{x})=\sum_{k=1}^{N} d_{k} K\left(\boldsymbol{x}, \boldsymbol{x}_{k}\right)$ 
holds. If

$L\left(\boldsymbol{x}, \boldsymbol{x}_{k}\right)=\sum_{n=0}^{\infty} \frac{2 n+1}{4 \pi R^{2}}\left(\frac{R}{r}\right)^{n+1} L_{n} P_{n}\left(\xi^{\mathrm{T}} \xi_{k}\right)$

is another kernel with

$L_{n}=0$ for $n>n^{\prime}$,

then

$(L * \bar{F})(\boldsymbol{x})=\sum_{k=1}^{N} d_{k} L\left(\boldsymbol{x}, \boldsymbol{x}_{k}\right)$.

The proof of this theorem can be found in Freeden et al. (1998a). Hence, we conclude that if the coefficients $d_{k}$ with $k=1, \ldots, N$ are known, they can be used to calculate any convolution of the signal $\bar{F}$ with kernel functions $L\left(\boldsymbol{x}, \boldsymbol{x}_{k}\right)$ as defined in Eqs. (38) and (39). Note, that the reproducing kernel (Eq. 30) is an example for the kernel $K\left(\boldsymbol{x}, \boldsymbol{x}_{k}\right)$ defined in Eqs. (35) and (36).

In vector notation, Eq. (40) can be rewritten as

$(L * \bar{F})(\boldsymbol{x})=\boldsymbol{l}(\boldsymbol{x})^{\mathrm{T}} \boldsymbol{d}$.

In order to derive a relation between the $N \times 1$ vectors $\boldsymbol{c}$ and $\boldsymbol{d}$, we equate the right-hand sides of Eqs. (33) and (34) and obtain, under the consideration of Eq. (41),

$$
\begin{aligned}
\boldsymbol{b}(\boldsymbol{x})^{\mathrm{T}}(\boldsymbol{c}-\boldsymbol{d}) & =\left(\boldsymbol{k}_{\mathrm{rep}}(\boldsymbol{x})-\boldsymbol{b}(\boldsymbol{x})\right)^{\mathrm{T}} \boldsymbol{d} \\
& =\Delta \boldsymbol{b}(\boldsymbol{x})^{\mathrm{T}} \boldsymbol{d}=(\Delta B * F)(\boldsymbol{x}),
\end{aligned}
$$

wherein $\Delta \boldsymbol{b}(\boldsymbol{x})$ denotes the $N \times 1$ vector of spherical base functions $\Delta B\left(\boldsymbol{x}, \boldsymbol{x}_{k}\right)$ with $k=1, \ldots, N . \Delta B\left(\boldsymbol{x}, \boldsymbol{x}_{k}\right)$ is defined analogously to Eq. (19) as series expansion with Legendre coefficients $\Delta B_{n}=1-B_{n}$.

Writing Eq. (42) for the $N$ position vectors $\boldsymbol{x}=\boldsymbol{x}_{l}$ with $l=1, \ldots, N$ of the admissible system $S_{N}$ (Eq. 16), the linear equation system

$\boldsymbol{X}(\boldsymbol{c}-\boldsymbol{d})=\Delta \boldsymbol{X} \boldsymbol{d}$

follows, wherein $\boldsymbol{X}$ and $\Delta \boldsymbol{X}$ are $N \times N$ matrices with entries $B\left(\boldsymbol{x}_{l}, \boldsymbol{x}_{k}\right)$ and $\Delta B\left(\boldsymbol{x}_{l}, \boldsymbol{x}_{k}\right)$, respectively. Due to $\operatorname{rank} \boldsymbol{X}=\bar{n}$ the solution

$\boldsymbol{c}=\left(\boldsymbol{I}-\boldsymbol{X}^{+} \Delta \boldsymbol{X}\right) \boldsymbol{d}$

can be derived with $\boldsymbol{X}^{+}$being the pseudoinverse of $\boldsymbol{X}$ and $\boldsymbol{I}$ the $N \times N$ unit matrix. Hence, if the vector $\boldsymbol{d}$ is known, the vector $c$ can be computed via Eq. (44). Since the deviation $\boldsymbol{c}-\boldsymbol{d}$ depends on the Legendre coefficients
$\Delta B_{n}$, the right-hand side of Eq. (18) is equivalent to the convolution $B * F$ only if $B\left(\boldsymbol{x}, \boldsymbol{x}_{k}\right)=K_{\text {rep }}\left(\boldsymbol{x}, \boldsymbol{x}_{k}\right)$.

In the case of a non-band-limited input signal $\bar{F}(\boldsymbol{x})$, the results derived above are only approximately valid. In order to demonstrate this, we introduce the decomposition $\bar{F}(\boldsymbol{x})=F(\boldsymbol{x})+S(\boldsymbol{x})$ with $F \in H_{0, \ldots, n^{\prime}}\left(\overline{\Omega_{R}^{\mathrm{ext}}}\right)$ and obtain with Eqs. (31) and (34)

$$
\begin{aligned}
\bar{F}(\boldsymbol{x})= & \left(K_{\mathrm{rep}} * F\right)(\boldsymbol{x})+S(\boldsymbol{x}) \\
= & \boldsymbol{k}_{\mathrm{rep}}(\boldsymbol{x})^{\mathrm{T}} \boldsymbol{d}+S(\boldsymbol{x}) .
\end{aligned}
$$

The influence of neglecting the non-stochastic signal $S(\boldsymbol{x})$ (omission error) on the determination of the coefficient vector $\boldsymbol{d}$ is known as the aliasing error; see, e.g., Kusche (2002).

\subsection{Multi-resolution representation}

As shown before, spherical base functions are related to spherical convolutions, i.e., the basic tool for lowand band-pass filtering processes. The fundamental idea of a multi-resolution representation (MRR) is to split a given input signal into a smoothed version and a number of detail signals by successive low-pass filtering; this procedure, which provides a sequence of signal approximations at different resolutions, is also known as multi-resolution analysis (MRA) (e.g., Mertins 1999). The detail signals are the spectral components or modules of the MRR because they are related to specific frequency bands.

In order to explain this procedure in more detail we identify the kernel $L\left(\boldsymbol{x}, \boldsymbol{x}_{k}\right)$, defined in Eqs. (38) and (39), with the spherical scaling function

$\Phi_{j}\left(\boldsymbol{x}, \boldsymbol{x}_{k}\right)=\sum_{n=0}^{n_{j}^{\prime}} \frac{2 n+1}{4 \pi R^{2}}\left(\frac{R}{r}\right)^{n+1} \Phi_{j ; n} P_{n}\left(\xi^{\mathrm{T}} \xi_{k}\right)$

of resolution level (scale) $j \in\{0, \ldots, J+1\}$; the scalars $\Phi_{j ; n}$ are the level-j Legendre coefficients of $\Phi_{j}\left(\boldsymbol{x}, \boldsymbol{x}_{k}\right)$ restricted to the condition $\Phi_{j ; n}=0$ for all $n>n_{j}^{\prime}$ according to Eq. (39) with $n^{\prime}=n_{j}^{\prime}$. The MRR states that a signal

$F_{j+1}(\boldsymbol{x})=\left(\Phi_{j+1} * F\right)(\boldsymbol{x})$

can be decomposed into the smoother version

$F_{j}(\boldsymbol{x})=\left(\Phi_{j} * F\right)(\boldsymbol{x})$

and the detail signal

$G_{j}(\boldsymbol{x})=\left(\Psi_{j} * F\right)(\boldsymbol{x})$ 
absorbing all the fine structures of $F_{j+1}(\boldsymbol{x})$ missing in $F_{j}(\boldsymbol{x}) ; \boldsymbol{x}=r \boldsymbol{\xi} \in \overline{\Omega_{R}^{\mathrm{ext}}}$. Hence, the MRR of the bandlimited input signal $F(\boldsymbol{x})=F_{J+1}(\boldsymbol{x})+\Delta F_{J+1}(\boldsymbol{x})$ can be written as

$F(\boldsymbol{x})=F_{j^{\prime}}(\boldsymbol{x})+\sum_{j=j^{\prime}}^{J} G_{j}(\boldsymbol{x})+\Delta F_{J+1}(\boldsymbol{x})$

with $j^{\prime} \in\{0, \ldots, J\}$.

If the scaling functions $\Phi_{j+1}\left(\boldsymbol{x}, \boldsymbol{x}_{k}\right)$ and $\Phi_{j}\left(\boldsymbol{x}, \boldsymbol{x}_{k}\right)$ act as low-pass filters, the spherical wavelet function

$\Psi_{j}\left(\boldsymbol{x}, \boldsymbol{x}_{k}\right)=\sum_{n=0}^{n_{j+1}^{\prime}} \frac{2 n+1}{4 \pi R^{2}}\left(\frac{R}{r}\right)^{n+1} \Psi_{j ; n} P_{n}\left(\xi^{\mathrm{T}} \xi_{k}\right)$

of level $j$ can be interpreted as a band-pass filter defined by its Legendre coefficients

$\Psi_{j ; n}=\Phi_{j+1 ; n}-\Phi_{j ; n}$

The application of Eq. (52) is called the linear wavelet approach, because it omits the computation of wavelet coefficients. Its alternative, i.e., the bilinear wavelet approach characterized by the computation of wavelet coefficients, is described in detail e.g., by Freeden et al. (1998a).

According to Eq. (41) the spherical convolution (Eq. 47) can be rewritten as

$F_{j+1}(\boldsymbol{x})=\boldsymbol{\phi}_{j+1}(\boldsymbol{x})^{\mathrm{T}} \boldsymbol{d}_{j}$

Herein

$\boldsymbol{\phi}_{j+1}(\boldsymbol{x})=\left(\Phi_{j+1}\left(\boldsymbol{x}, \boldsymbol{x}_{1}^{j}\right), \Phi_{j+1}\left(\boldsymbol{x}, \boldsymbol{x}_{2}^{j}\right), \ldots, \Phi_{j+1}\left(\boldsymbol{x}, \boldsymbol{x}_{N_{j}}^{j}\right)\right)^{\mathrm{T}}$

and

$\boldsymbol{d}_{j}=\left(d_{j ; 1}, d_{j ; 2}, \ldots, d_{j ; N_{j}}\right)^{\mathrm{T}}$

are $N_{j} \times 1$ vectors of scaling function values $\Phi_{j+1}\left(\boldsymbol{x}, \boldsymbol{x}_{k}^{j}\right)$ and level-j scaling coefficients $d_{j: k}$. Recall that the points $\boldsymbol{x}_{k}^{j}$ constitute an admissible system $S_{N_{j}}=\left\{\boldsymbol{x}_{k}^{j} \mid k=\right.$ $\left.1, \ldots, N_{j}\right\}$ of level $j$. Since the spherical wavelet function (Eq. 51) fulfills Eq. (39), Eq. (49) can be rewritten as

$G_{j}(\boldsymbol{x})=\boldsymbol{\psi}_{j}(\boldsymbol{x})^{\mathrm{T}} \boldsymbol{d}_{j}$,

wherein

$\psi_{j}(\boldsymbol{x})=\left(\Psi_{j}\left(\boldsymbol{x}, \boldsymbol{x}_{1}^{j}\right), \Psi_{j}\left(\boldsymbol{x}, \boldsymbol{x}_{2}^{j}\right), \ldots, \Psi_{j}\left(\boldsymbol{x}, \boldsymbol{x}_{N_{j}}^{j}\right)\right)^{\mathrm{T}}$ means an $N_{j} \times 1$ vector of wavelet function values $\Psi_{j}\left(\boldsymbol{x}, \boldsymbol{x}_{k}^{j}\right)$. Writing Eq. (53) for the next lower level $j$ yields

$F_{j}(\boldsymbol{x})=\boldsymbol{\phi}_{j}(\boldsymbol{x})^{\mathrm{T}} \boldsymbol{d}_{j-1}$

with $\boldsymbol{\phi}_{j}(\boldsymbol{x})$ and $\boldsymbol{d}_{j-1}$ being the $N_{j-1} \times 1$ vectors of scaling function values $\boldsymbol{\phi}_{j}\left(\boldsymbol{x}, \boldsymbol{x}_{k}^{j-1}\right)$ and level-(j-1) scaling coefficients $d_{j-1 ; k}$ related to the $N_{j-1}$ points $\boldsymbol{x}_{k}^{j-1}$ of a level- $(j-1)$ admissible system $S_{N_{j-1}}=\left\{x_{k}^{j-1} \mid k=1, \ldots\right.$, $\left.N_{j-1}\right\}$.

Fortunately, the linear relation

$\boldsymbol{d}_{j-1}=\boldsymbol{W}_{j-1} \boldsymbol{K}_{j} \boldsymbol{d}_{j}$

allows us to connect the scaling coefficients of subsequent levels, i.e., all scaling coefficient vectors $\boldsymbol{d}_{j-1}$ with $j=j^{\prime}+1, \ldots, J$ are computable successively (Freeden 1999). In Eq. (59) $\boldsymbol{K}_{j}$ means an $N_{j-1} \times N_{j}$ matrix with entries $K_{\text {rep }}\left(\boldsymbol{x}_{k}^{j-1}, \boldsymbol{x}_{l}^{j}\right)$ for $k=1, \ldots, N_{j-1}$ and $l=$ $1, \ldots, N_{j} ; \boldsymbol{W}_{j-1}=\operatorname{diag}\left(w_{1}^{j-1}, w_{2}^{j-1}, \ldots, w_{N_{j-1}}^{j-1}\right)$ is an $N_{j-1} \times$ $N_{j-1}$ diagonal matrix of the integration weights $w_{k}^{j-1}$ associated with the points $\boldsymbol{x}_{k}^{j-1}$ of the admissible system $S_{N_{j-1}}$; an example of integration weights will be given in Eq. (68).

According to Eq. (40) the spherical convolution in Eq. (48) can also be evaluated by means of the scaling vector $\boldsymbol{d}_{j}$ used in Eqs. (53) and (56). However, this procedure would have the drawback that in each decomposition step, the same admissible system is used, although coarser structures are representable by fewer terms than finer structures.

As shown in Fig. 1, the procedure for constituting the MRR (Eq. 50), starts with the calculation of the vector $\boldsymbol{d}_{J}$ in the initial step. Since the inequality $N_{j-1}<N_{j}$ usually holds, the $N_{j-1} \times N_{j}$ transformation matrix $\boldsymbol{K}_{j}$ in Eq. (59) effects a downsampling process as the key point of the pyramid algorithm (filter bank scheme).

Finally, we have to focus on the last term $\Delta F_{J+1}(\boldsymbol{x})$ of the MRR (Eq. 50). Considering Eqs. (31) and (47) for $j=J$ we obtain

$$
\begin{aligned}
\Delta F_{J+1}(\boldsymbol{x}) & =F(\boldsymbol{x})-F_{J+1}(\boldsymbol{x}) \\
& =\left(\left(K_{\mathrm{rep}}-\Phi_{J+1}\right) * F\right)(\boldsymbol{x}) \\
& =\left(\Delta \Phi_{J+1} * F\right)(\boldsymbol{x})=\Delta \boldsymbol{\phi}_{J+1}(\boldsymbol{x})^{\mathrm{T}} \boldsymbol{d}_{J}
\end{aligned}
$$

according to Eq. (42). As mentioned in the context of Eq. (44), the magnitude of Eq. (60) depends on the Legendre coefficients $\Delta \Phi_{J+1 ; n}=1-\Phi_{J+1 ; n}$ of the spher- 


$$
\begin{aligned}
& \boldsymbol{y} \rightarrow \boldsymbol{d}_{J} \stackrel{\downarrow}{\rightarrow} \boldsymbol{d}_{J-1} \stackrel{\downarrow}{\rightarrow} \cdots \stackrel{\downarrow}{\rightarrow} \boldsymbol{d}_{j} \stackrel{\downarrow}{\rightarrow} \boldsymbol{d}_{j-1} \stackrel{\downarrow}{\rightarrow} \cdots \stackrel{\downarrow}{\rightarrow} \boldsymbol{d}_{j^{\prime}} \\
& \downarrow \quad \downarrow \quad \downarrow \quad \downarrow \quad \downarrow \\
& G_{J}+G_{J-1}+\cdots+G_{j}+G_{j-1}+\cdots+G_{j^{\prime}}+F_{j^{\prime}}+\Delta F_{J+1}=F
\end{aligned}
$$

Fig. 1 Filter bank of the multi-resolution representation (MRR) using wavelets. “ $\stackrel{\downarrow}{\rightarrow}$ ” means a symbol for downsampling, e.g., from level $j$ to level $j-1$ by a factor $N_{j} / N_{j-1}$. The transformation from
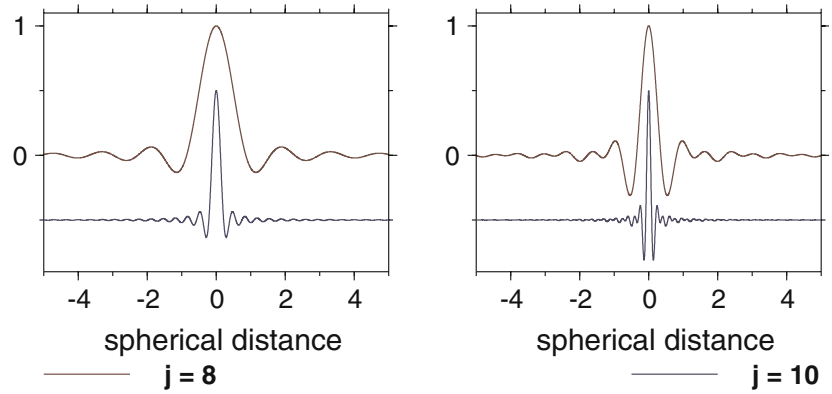

Fig. 2 Selected Shannon scaling functions (left panel) and wavelets (right panel) of levels $j=8$ and $j=10$ with respect to the spherical distance $\alpha$ (degrees); ordinate values are normed to 1 , the functions for $j=10$ are shifted by -0.5 along the ordinate axis

ical base function $\Delta \Phi_{J+1}\left(\boldsymbol{x}, \boldsymbol{x}_{k}\right)$. Further considerations about this statement are given by Schmidt et al. (2005c).

The procedure described before can be applied to global and regional (local) data sets. In the latter case, the localization feature of the spherical base functions reduces undesired boundary effects. This item will be discussed in more detail in Sect. 3.1. Before concluding this section, we would like to state some examples for scaling functions and wavelets.

Since there exist many examples for spherical wavelets in the literature (e.g., Freeden 1999), we restrict ourselves here to only four examples in dyadic representation, i.e. the relation $n_{j}^{\prime}=2^{j}-1$ holds in Eq. (46). We list the Legendre coefficients of the scaling functions; the related wavelets are computable via Eqs. (51) and (52).

1. The Shannon scaling function (Fig. 2; note that this figure and all the following figures are colored in the online version of this paper) possesses the simplest representation, i.e. the Legendre coefficients $\Phi_{j ; n}=: \Phi_{j ; n}^{\text {Sha }}$ are given by

$$
\Phi_{j ; n}^{\text {Sha }}=\left\{\begin{array}{ll}
1 & \text { if } n \in\left[0,2^{j}\right) \\
0 & \text { else }
\end{array} .\right.
$$

the observation vector $\boldsymbol{y}$ into the scaling coefficient vector $\boldsymbol{d}_{J}$ is explained in Sect. 3. The elements of the vectors $\boldsymbol{d}_{j}$, as well as the signals $G_{j}, F_{j^{\prime}}$ and $\Delta F_{J+1}$, are computable via Eqs. (56), (58), (59) and (60)
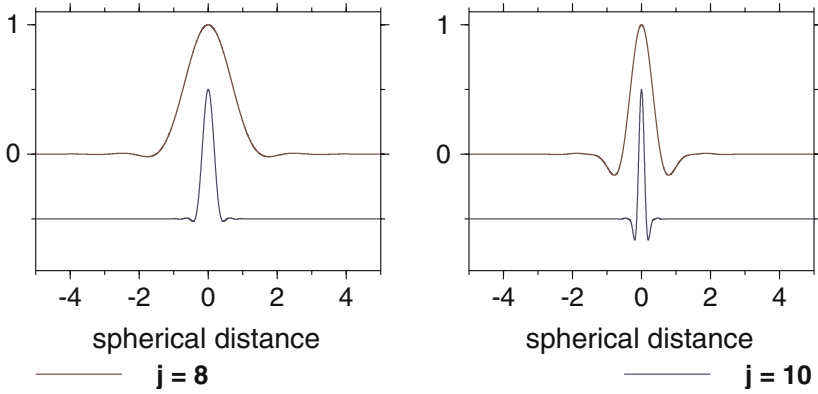

Fig. 3 Selected CuP scaling functions (left panel) and wavelets (right panel) of levels $j=8$ and $j=10$ with respect to the spherical distance $\alpha$ (degrees); ordinate values are normed to 1 , the functions for $j=10$ are shifted by -0.5 along the ordinate axis
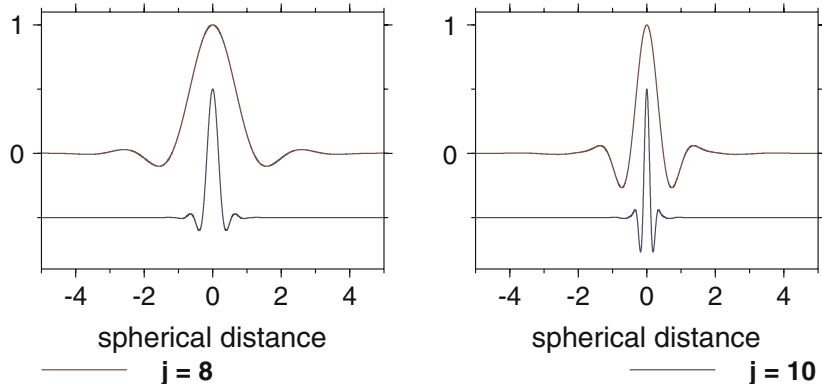

Fig. 4 Selected Blackman scaling functions (left panel) and wavelets (right panel) of levels $j=8$ and $j=10$ with respect to the spherical distance $\alpha$ (degrees); ordinate values are normed to 1 , the functions for $j=10$ are shifted by -0.5 along the ordinate axis

2. The CuP scaling function (Fig. 3) is motivated by smoothing the decay of the power spectrum by a cubic polynomial (CuP). Its Legendre coefficients $\Phi_{j ; n}=: \Phi_{j ; n}^{\mathrm{CuP}}$ are given by

$\Phi_{j ; n}^{\mathrm{CuP}}=\left\{\begin{array}{ll}\left(1-2^{-j} n\right)^{2}\left(1+2^{-j+1} n\right) & \text { if } n \in\left[0,2^{j}\right) \\ 0 & \text { else }\end{array}\right.$.

3. The Blackman scaling function (Fig. 4) is derived from the Blackman window, which is often used in classical signal analysis (e.g., Mertins (1999)). The 

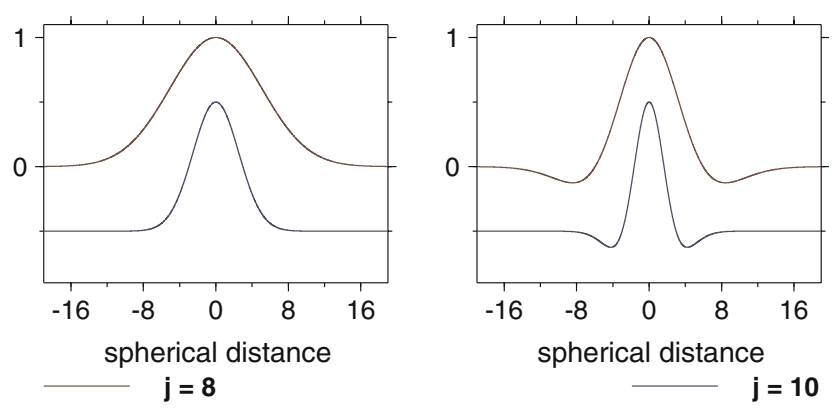

Fig. 5 Selected Bernstein scaling functions (left panel) and wavelets (right panel) of levels $j=8$ and $j=10$ with respect to the spherical distance $\alpha$ (degrees); ordinate values are normed to 1 , the functions for $j=10$ are shifted by -0.5 along the ordinate axis, axis of abscissae is zoomed out in comparison with Figs. (2) to (4)

Legendre coefficients $\Phi_{j ; n}=: \Phi_{j ; n}^{\mathrm{Bla}}$ are defined as

$$
\Phi_{j ; n}^{\mathrm{Bla}}= \begin{cases}1 & \text { if } n \in\left[0,2^{j-1}\right) \\ A_{j}(n) & \text { if } n \in\left[2^{j-1}, 2^{j}\right) \\ 0 & \text { else }\end{cases}
$$

wherein

$$
A_{j}(n)=\frac{21}{50}-\frac{1}{2} \cos \left(\frac{2 \pi n}{2^{j}}\right)+\frac{2}{25} \cos \left(\frac{4 \pi n}{2^{j}}\right) .
$$

As can be seen from Figs. (2), (3) and (4), the Blackman scaling function means some kind of compromise between the Shannon and the CuP kernel, i.e., it possesses the appealing feature (depending on the application) that the low-frequency signal content remains unfiltered. A more general version of the Blackman scaling function will be defined in Eq. (80).

4. The Bernstein scaling function (Fig. 5) possesses the favorable property of a closed-form representation, which makes it unique among all of the band-limited scaling functions until now. Its Legendre coefficients $\Phi_{j ; n}=: \Phi_{j ; n}^{\mathrm{Ber}}$ are given by

$$
\Phi_{j ; n}^{\mathrm{Ber}}=\left\{\begin{array}{ll}
\frac{\left(2^{j}\right) !\left(2^{j}-1\right) !}{\left(2^{j}-n-1\right) !\left(2^{j}+n\right) !} & \text { if } n \in\left[0,2^{j}\right) \\
0 & \text { else }
\end{array} .\right.
$$

Its closed-form representation reads (Fengler et al. 2005)

$$
\Phi_{j}^{\mathrm{Ber}}(\boldsymbol{\xi}, \boldsymbol{\eta})=\frac{2^{j-2}}{\pi}\left(\frac{1+\xi^{\mathrm{T}} \boldsymbol{\eta}}{2}\right)^{2^{j}-1}, \quad \xi, \boldsymbol{\eta} \in \Omega
$$

Finally, we also want to mention the Gaussian kernel, which is often used to smooth the input data (Jekeli
1981), e.g., for computing time-dependent gravity fields from GRACE measurements; see e.g., Wahr et al. 1998, Swenson and Wahr (2002) as well as Sect. 4.4 of this paper.

\section{Model parameter determination}

\subsection{Numerical integration}

In "classical wavelet analysis", the computation of scaling and wavelet coefficients is performed by discretizing the spherical convolution integrals by means of quadrature rules; see Freeden et al. (1998a) and Freeden (1999). FFT-based algorithms allow the rapid evaluation globally by involving tensor-product-based integration grids.

A well-known example of a level- $j$ admissible point system $S_{N_{j}}$ (Eq. 16) is the standard longitude-latitude grid, i.e. the $N_{j}$ grid points $\boldsymbol{x}_{k}^{j}, k=1, \ldots, N_{j}$ on the sphere $\Omega_{R}$, with

$\boldsymbol{x}_{k}^{j}=: \boldsymbol{x}_{i, l}^{j}=R\left(\cos \beta_{l}^{j} \cos \lambda_{i}^{j}, \cos \beta_{l}^{j} \sin \lambda_{i}^{j}, \sin \beta_{l}^{j}\right)^{\mathrm{T}}$

defined by the discrete spherical coordinates $\lambda_{i}^{j}=i \pi / L_{j}$ and $\beta_{l}^{j}=-\pi / 2+l \pi /\left(2 L_{j}\right)$ in longitude $\lambda$ and latitude $\beta$ with $i=0, \ldots, 2 L_{j}-1, l=0, \ldots, 2 L_{j}$ and $N_{j}=\left(2 L_{j}+\right.$ 1) $\cdot 2 L_{j}$. The corresponding integration weights

$$
\begin{aligned}
w_{k}^{j} & =: w_{i, l}^{j} \\
& =\frac{2 \pi R^{2}}{L_{j}^{2}} \sin \left(\frac{l \pi}{2 L_{j}}\right) \sum_{k=0}^{L_{j}-1} \frac{1}{2 k+1} \sin \left(\frac{(2 k+1) l \pi}{2 L_{j}}\right)
\end{aligned}
$$

are merely latitude-dependent (Driscoll and Healy 1994). For other types of grids such as spiral, icosahedral, Reuter, Brandt, or Corput-Halton grids (e.g., Freeden 1999) or irregularly distributed points, the fast computation can be performed adaptively by aid of spherical panel clustering (Freeden et al. 1998b). If the function (input signal) $F$ is given at the positions $\boldsymbol{x}_{k}^{J}$ of the admissible system $S_{N_{J}}$ of the highest level $J$, the components $d_{J ; k}$ of the coefficient vector $\boldsymbol{d}_{J}$ (Eq. 55) with $k=1, \ldots, N_{J}$ are computable from (e.g. Freeden et al., 1998a)

$d_{J ; k}=w_{k}^{J} F\left(\boldsymbol{x}_{k}^{J}\right)$

The successive application of Eq. (59) yields all remaining scaling coefficient vectors shown in Fig. 1; therein the observation vector $\boldsymbol{y}$ is defined as $\boldsymbol{y}=\left(F\left(\boldsymbol{x}_{1}^{J}\right)\right.$, 
$\left.F\left(\boldsymbol{x}_{2}^{J}\right), \ldots, F\left(\boldsymbol{x}_{N_{J}}^{J}\right)\right)^{\mathrm{T}}$. Note, that we do not consider measurement errors of the input data in Eq. (69).

Moreover, numerical integration has the advantage that no (ill-conditioned) linear system has to be solved, and, more importantly in case of regional solutions, the approximations show nearly no blurring due to the Gibbs phenomenon. However, until now, the drawback of numerical integration is that the classical spherical wavelet analysis is bounded to the sphere or at least to regular surfaces or subdomains of it. This is due to the lack of appropriate cubature formulae to the whole exterior of the sphere $\Omega_{R}$.

This problem can be overcome by applying the approaches presented before in Sects. 2.2, 2.3 and 2.4 successively, i.e., the representation (Eq. 24) in spherical base functions (splines) (Eq. 19) can be used to consider the non-sphericity of the satellite orbits and the spherical wavelets (Eq. 51) for providing the multi-resolution properties on the sphere (Fengler et al.2004a). An alternative approach is given in Fengler et al. (2006a), where multi-resolutions are defined via cascades of spline approximations. Nevertheless, spherical wavelets have probably their greatest impact in the solution of inverse problems by aid of regularization wavelets; e.g., Tikhonov regularization wavelets are treated in Freeden (1999), Fengler et al. (2004a) and Schneider (1996).

To be more specific, here we investigate an example that is discussed in more detail in Sect. 4.2, namely the regional recovery of the Earth's gravity field from Faye gravity anomalies given within the Colombian region (Fig. 13). For better comparison, we computed reconstructions of the given data using all four wavelet types introduced at the end of Sect. 2.4. We observe that the reconstruction depends on the shape of the kernel function used.

From Figs. 2, 3 and 4 we note that due to the spatial localization the Shannon, Blackman and CuP kernels cover approximately only a spherical cap of two degrees at level $j=8$, which decreases nearly by half if the level is increased by one, e.g., we note from Figs. 2, 3 and 4 a support diameter of about 0.5 degree for level $j=10$. This strong spatial localization effectively detects the small terrain-correlated details in the Faye anomaly data set.

However, we also note small differences between the approximations of the $\mathrm{CuP}$ and Blackman reconstruction process and the Shannon approximations. From Fig. 2, we note many ripples of the Shannon kernel that introduce a characteristic blurring into the approximations given in Fig. 6; see particularly the panels of the detail signals $G_{9}(\boldsymbol{x})$ and $G_{10}(\boldsymbol{x})$. This effect is also known as spectral ringing from classical Fourier theory.
Interestingly, Figs. 7 and 8 show that this ringing can be minimized or even avoided by taking a smooth kernel into account. Optimal smoothness in the sense that the kernel shows no (micro-)oscillations is guaranteed by the Bernstein kernel (Fig. 9). However, as Fig. 5 shows, the spatial localization increases slower than that of the other three examples. The consequence is a larger support of the kernel leading to boundary effects. Such boundary effects can occur as ripples or as a sudden decay to the boundary if the kernel has a support that is much greater than the regional details to be investigated. Since boundary effects are of special interest for the detail signals of the lower levels, they disappear when increasing the level (not shown here).

\subsection{Parameter estimation}

Following Eqs. (24) and (34), the function $F(\boldsymbol{x})$ can generally be modeled as

$F(\boldsymbol{x})=\boldsymbol{a}(\boldsymbol{x})^{\mathrm{T}} \boldsymbol{\beta}$,

wherein the $N \times 1$ vector $\boldsymbol{a}(\boldsymbol{x}) \in\left\{\boldsymbol{b}(\boldsymbol{x}), \boldsymbol{k}_{\mathrm{rep}}(\boldsymbol{x})\right\}$ contains the values of the spherical base functions.

In order to estimate the unknown $N \times 1$ coefficient vector $\boldsymbol{\beta}=\boldsymbol{c}$ for $\boldsymbol{a}(\boldsymbol{x})=\boldsymbol{b}(\boldsymbol{x})$ or $\boldsymbol{\beta}=\boldsymbol{d}$ for $\boldsymbol{a}(\boldsymbol{x})=$ $\boldsymbol{k}_{\text {rep }}(\boldsymbol{x})$, we need values $F\left(\boldsymbol{x}_{p}\right)=: F_{p}$ of the function $F(\boldsymbol{x})$ in altogether $P$ discrete observation points $\boldsymbol{x}_{p} \in \overline{\Omega_{R}^{\mathrm{ext}}}$ with $p=1, \ldots, P$ and $P \geq N$.

Since geodetic measurements $y\left(\boldsymbol{x}_{p}\right)=: y_{p}$ are always affected by measurement errors $e_{p}:=e\left(\boldsymbol{x}_{p}\right)$, i.e. $F_{p}=$ $y_{p}+e_{p}$, Eq. (70) can be rewritten as

$y_{p}+e_{p}=\boldsymbol{a}_{p}^{\mathrm{T}} \boldsymbol{\beta}$

with $\boldsymbol{a}\left(\boldsymbol{x}_{p}\right)=: \boldsymbol{a}_{p}$. Introducing the $P \times 1$ vectors $\boldsymbol{y}=$ $\left(y_{1}, y_{2}, \ldots, y_{P}\right)^{\mathrm{T}}$ and $\boldsymbol{e}=\left(e_{1}, e_{2}, \ldots, e_{P}\right)^{\mathrm{T}}$ of the observations and the measurement errors, respectively, the $P \times N$ coefficient matrix $\boldsymbol{A}=\left(\boldsymbol{a}_{1}, \boldsymbol{a}_{2}, \ldots, \boldsymbol{a}_{P}\right)^{\mathrm{T}}$ and the $P \times P$ covariance matrix $D(y)$ of the observations, the linear model

$\boldsymbol{y}+\boldsymbol{e}=\boldsymbol{A} \boldsymbol{\beta} \quad$ with $\quad D(\boldsymbol{y})=\sigma_{y}^{2} \boldsymbol{P}_{y}^{-1}$

is established. Herein $\sigma_{y}^{2}$ and $\boldsymbol{P}_{y}$ are denoted as the variance factor and the weight matrix, respectively; see Koch (1999).

Analogous to the matrix $\boldsymbol{H}$ (Eq. 14) and depending on the distribution of the observation sites, the matrix $\boldsymbol{A}$ is at most of rank $\bar{n}$, i.e., a rank deficiency of at least $N-\bar{n}$ exists. In the following, however, we will assume that $\operatorname{rank} \boldsymbol{A}=\bar{n}$ holds. 


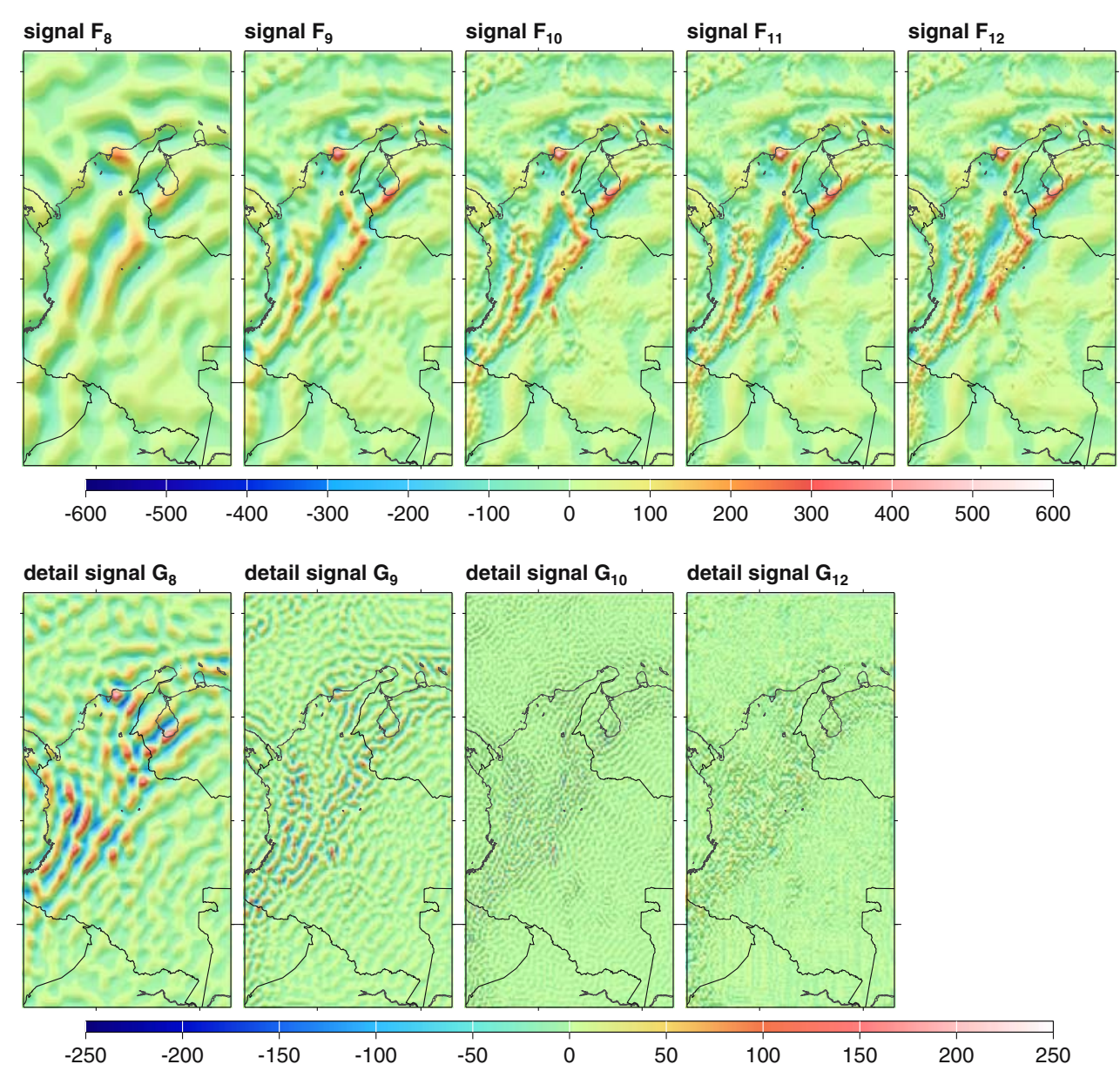

Fig. 6 Approximations of Faye anomalies in South America using Shannon scaling functions and wavelets of levels $j=8, \ldots, 12(\mathrm{mGal})$

Besides the rank deficiency problem, the normal equation system following from the application of the least-squares method to the linear model (Eq. 72) might be ill-conditioned. If we, e.g., want to compute the gravity field at the Earth's surface only from satellite data, this inverse problem requires regularization. Here, we solve both problems, the rank deficiency and the regularization, together.

If we assume that prior information for the expectation vector $E(\boldsymbol{\beta})=\boldsymbol{\mu}_{\beta}$ and the covariance matrix $D(\boldsymbol{\beta})=\boldsymbol{P}_{\beta}^{-1}$ of the vector $\boldsymbol{\beta}$ is available, the additional linear model

$\boldsymbol{\mu}_{\beta}+\boldsymbol{e}_{\beta}=\boldsymbol{\beta} \quad$ with $\quad D\left(\boldsymbol{\mu}_{\beta}\right)=\sigma_{\beta}^{2} \boldsymbol{P}_{\beta}^{-1}$

can be formulated. Herein, $\boldsymbol{e}_{\beta}$ is defined as the error vector of the prior information and $\sigma_{\beta}^{2}$ the corresponding unknown variance factor. The combination of the two models (Eqs. 72 and 73) gives an extended linear model with unknown variance components $\sigma_{y}^{2}$ and $\sigma_{\beta}^{2}$, namely

$$
\begin{aligned}
{\left[\begin{array}{c}
\boldsymbol{y} \\
\boldsymbol{\mu}_{\beta}
\end{array}\right]+\left[\begin{array}{c}
\boldsymbol{e} \\
\boldsymbol{e}_{\beta}
\end{array}\right]=\left[\begin{array}{c}
\boldsymbol{A} \\
\boldsymbol{I}
\end{array}\right] \boldsymbol{\beta} \quad \text { with } } \\
D\left(\left[\begin{array}{c}
\boldsymbol{y} \\
\boldsymbol{\mu}_{\beta}
\end{array}\right]\right)=\sigma_{y}^{2}\left[\begin{array}{cc}
\boldsymbol{P}_{y}^{-1} & \mathbf{0} \\
\mathbf{0} & \mathbf{0}
\end{array}\right]+\sigma_{\beta}^{2}\left[\begin{array}{cc}
\mathbf{0} & \mathbf{0} \\
\mathbf{0} & \boldsymbol{P}_{\beta}^{-1}
\end{array}\right] .
\end{aligned}
$$

The application of the least-squares method to this model yields the normal equations

$$
\left(\boldsymbol{A}^{\mathrm{T}} \boldsymbol{P}_{y} \boldsymbol{A}+\lambda \boldsymbol{P}_{\beta}\right) \widehat{\boldsymbol{\beta}}=\boldsymbol{A}^{\mathrm{T}} \boldsymbol{P}_{y} \boldsymbol{y}+\lambda \boldsymbol{P}_{\beta} \boldsymbol{\mu}_{\beta}
$$

with the regularization parameter $\lambda:=\sigma_{y}^{2} / \sigma_{\beta}^{2}$. The method of estimating variance components (e.g., Koch 1999) allows the computation of an estimator of $\lambda$ by means of

$\widehat{\sigma}_{y}^{2}=\left(\widehat{\boldsymbol{e}}^{\mathrm{T}} \boldsymbol{P}_{y} \widehat{\boldsymbol{e}}\right) / r_{y}$,

$\widehat{\sigma}_{\beta}^{2}=\left(\widehat{\boldsymbol{e}}_{\beta}^{\mathrm{T}} \boldsymbol{P}_{\beta} \widehat{\boldsymbol{e}}_{\beta}\right) / r_{\beta}$. 


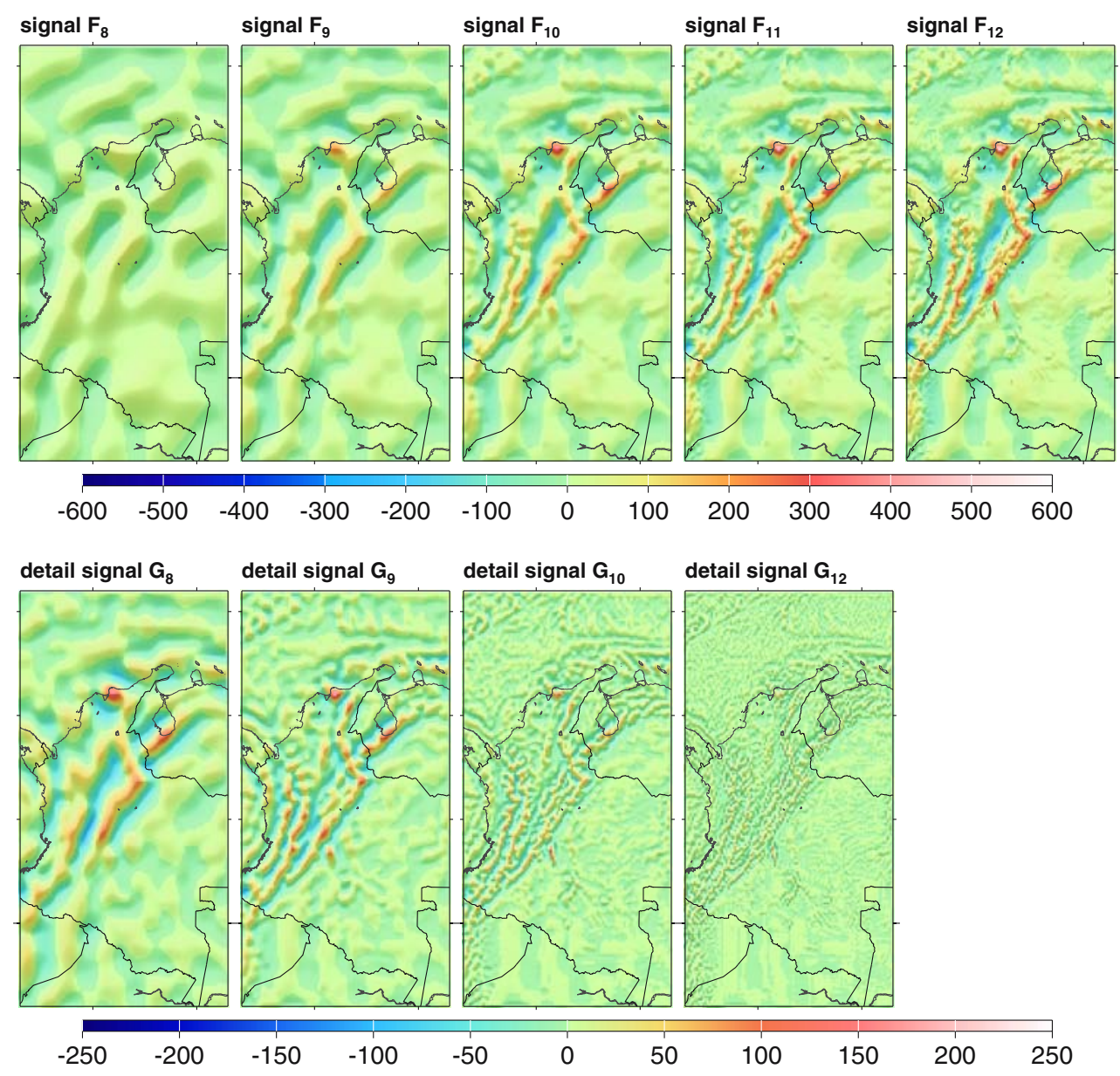

Fig. 7 Approximations of Faye anomalies in South America using CuP scaling functions and wavelets of levels $j=8, \ldots, 12$ (mGal)

Herein $\widehat{\boldsymbol{e}}$ and $\widehat{\boldsymbol{e}}_{\beta}$ denote the residual vectors as well as $r_{y}$ and $r_{\beta}$ the partial redundancies, i.e., the contributions of the observations and the prior information to the total redundancy $r=r_{y}+r_{\beta}=P$ of the linear model (Eq. 74).

Thus, the unbiased estimator

$$
\widehat{\boldsymbol{\beta}}=\left(\boldsymbol{A}^{\mathrm{T}} \boldsymbol{P}_{y} \boldsymbol{A}+\lambda \boldsymbol{P}_{\beta}\right)^{-1}\left(\boldsymbol{A}^{\mathrm{T}} \boldsymbol{P}_{y} \boldsymbol{y}+\lambda \boldsymbol{P}_{\beta} \boldsymbol{\mu}_{\beta}\right)
$$

with the covariance matrix

$$
D(\widehat{\boldsymbol{\beta}})=\sigma_{y}^{2}\left(\boldsymbol{A}^{\mathrm{T}} \boldsymbol{P}_{y} \boldsymbol{A}+\lambda \boldsymbol{P}_{\beta}\right)^{-1}
$$

is obtained from Eq. (75) if the variance component estimation converges. In the case $\boldsymbol{\beta}=\boldsymbol{d}=\boldsymbol{d}_{J}$, the estimator $\widehat{\boldsymbol{d}}_{J}$ is the starting point of the MRR as mentioned in the context of Fig. 1. Note, that the covariance matrices of all coefficient vectors $\boldsymbol{d}_{j}$ and all detail signals $G_{j}(\boldsymbol{x})$ are calculable from the covariance matrix $D(\widehat{\boldsymbol{\beta}})$ applying the law of error propagation.
The procedure described here allows the combination of different kinds of measurements, e.g., gravity anomalies and potential values. Then additional operators, like the Stokes operator, have to be considered in the vector $\boldsymbol{a}_{p}$ of Eq. (71). In opposite to that the numerical integration, discussed in the previous subsection, is restricted to one single type of observation, e.g., gravity anomalies (e.g., Torge 2001).

A theoretical study on the condition number of the (regularized) normal matrix in Eq. (75) and its dependency from the chosen point system and the base function type, has been conducted by Kusche (2002).

One also may desire an estimation of the detail signals $G_{j}(\boldsymbol{x})$ for $j=j^{\prime}, \ldots, J$ directly from Eq. (50). However, it follows from Eq. (52), that e.g., in the Blackman case (Eqs. 63 and 64) the wavelet functions $\Psi_{j}^{\mathrm{Bla}}\left(\boldsymbol{x}, \boldsymbol{x}_{k}\right)$ and $\Psi_{j+1}^{\text {Bla }}\left(\boldsymbol{x}, \boldsymbol{x}_{k}\right)$ of two consecutive levels $j$ and $j+1$ overlap in the spectral domain. Thus, according to Eq. (49) the corresponding detail signals $G_{j}(\boldsymbol{x})$ and $G_{j+1}(\boldsymbol{x})$ are 


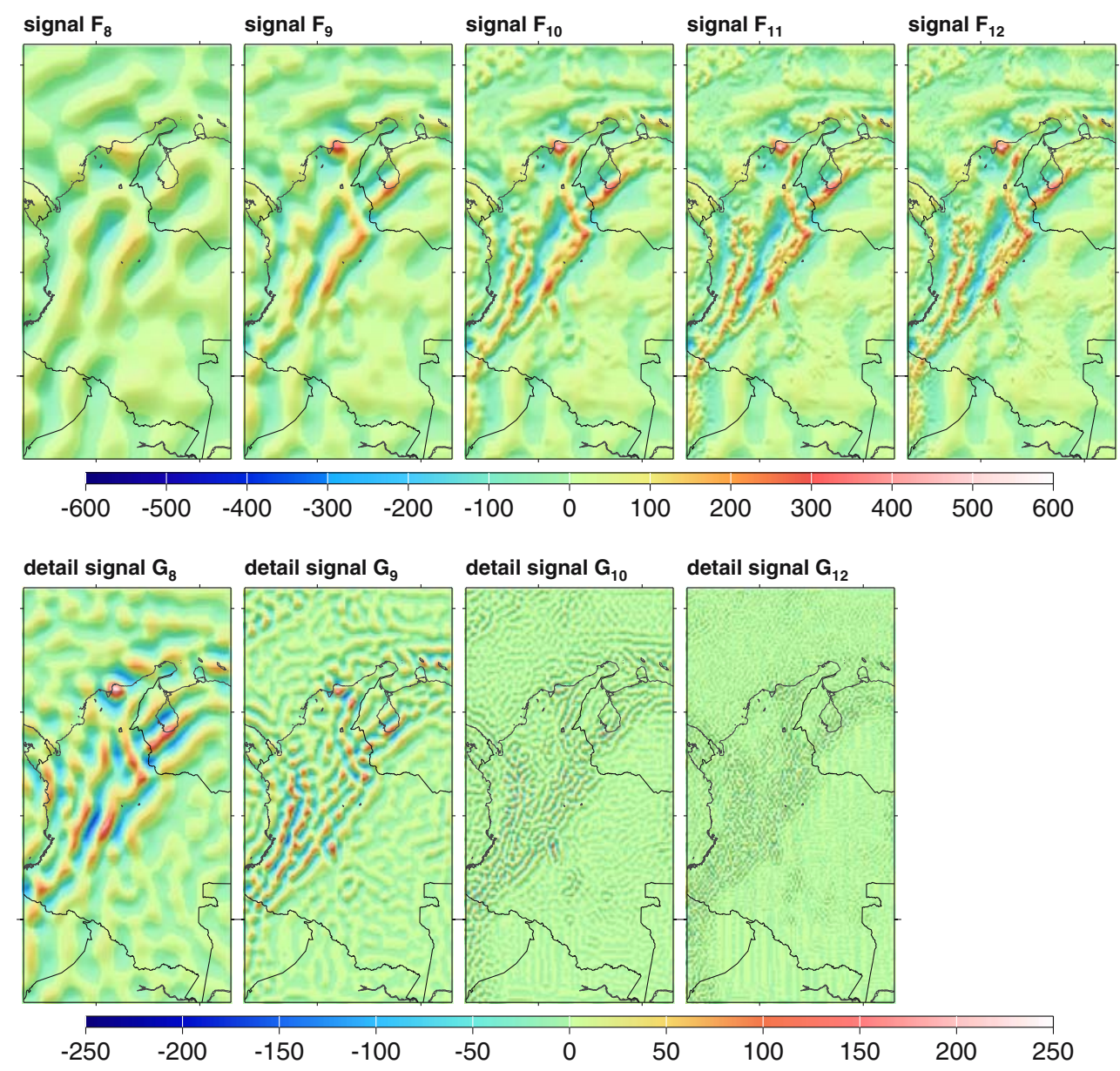

Fig. 8 Approximations of Faye anomalies in South America using Blackman scaling functions and wavelets of levels $j=8, \ldots, 12$ (mGal)

not independent of each other and cannot be estimated together without introducing additional constraints.

\section{High-resolution gravity field modeling}

In this section, we present four different regional geopotential models based on spherical wavelet or spline theory, respectively, and computed from measurements of two space missions, namely CHAMP and GRACE. Furthermore, the second example presents a combination of satellite and surface data.

\subsection{CHAMP-only model}

The energy balance approach and its application to low-Earth-orbiting (LEO) satellites like CHAMP and GRACE goes back to the 1960s (e.g., Bjerhammer 1967) and was rediscovered by Jekeli (1999), van Loon and
Kusche (2005), )) and many other authors. An extensive treatise of this topic can be found in Han (2003).

Here, kinematic CHAMP orbits, kindly provided by D. Švehla from the Technical University of Munich and covering a 2-year time-span, were converted via the energy balance approach into residual geopotential values $\delta V=V-\bar{V}$, using the accelerometry and altitude data products from GFZ (GeoForschungsZentrum) Potsdam; for details see van Loon and Kusche (2005) as well as Schmidt et al. (2005b).

The expression "residual" means that we study the geopotential $V$ with respect to EGM96 (Lemonie et al. 1998) complete to degree and order $n=120$ as the reference model $\bar{V}$. Since at a certain time $t=t_{p}$ CHAMP is located at position $\boldsymbol{x}\left(t_{p}\right)=\boldsymbol{x}_{p}$ along its orbit, the residual geopotential value $\delta V\left(\boldsymbol{x}\left(t_{p}\right)\right)=\delta V\left(\boldsymbol{x}_{p}\right)=: \delta V_{p}$ corresponds to the CHAMP in situ potential observation $y_{p}$ as introduced in Eq. (71). Figure 10 shows the input data set of observations $\delta V_{p}$ over the northern part of South America. 


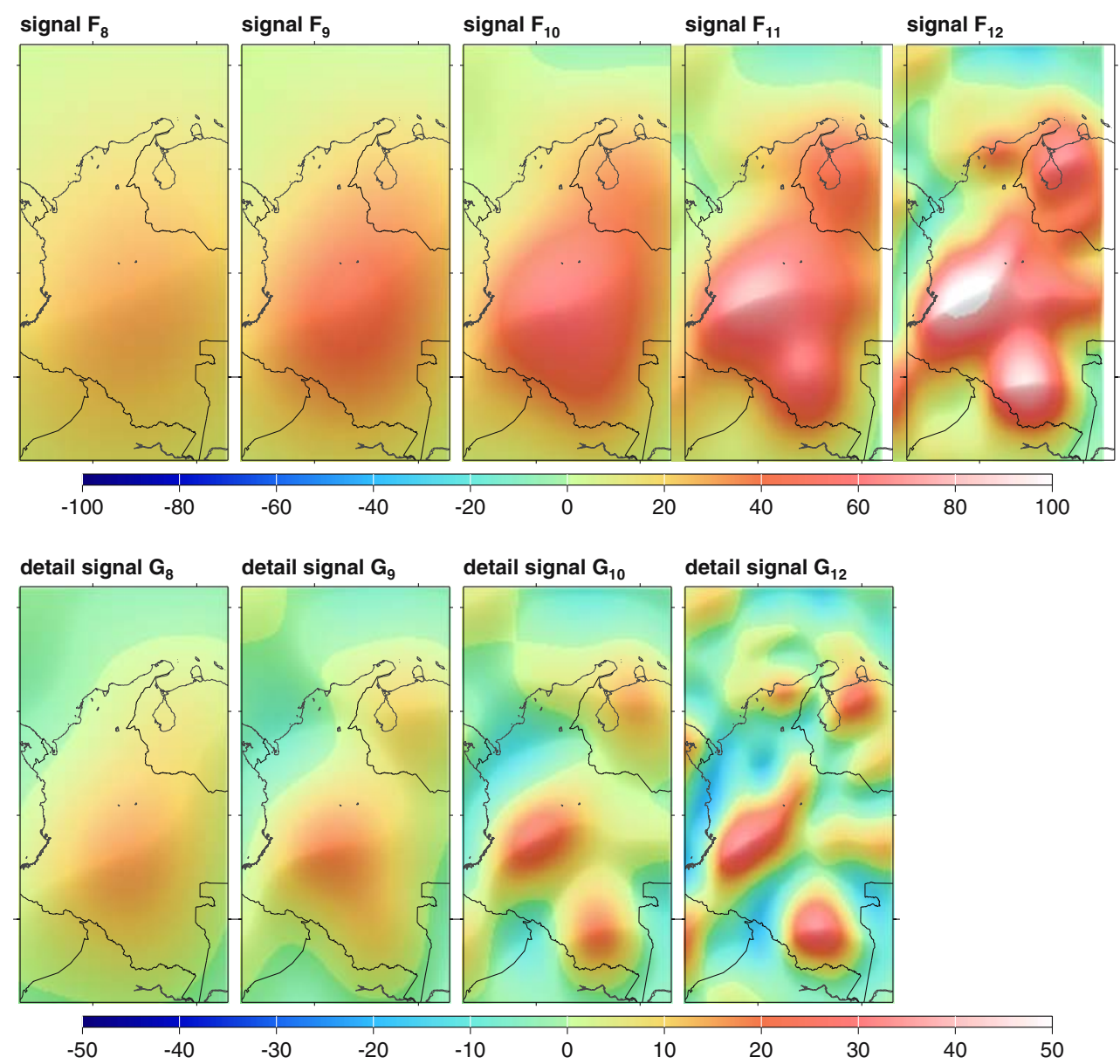

Fig. 9 Approximations of Faye anomalies in South America using Bernstein scaling functions and wavelets of levels $j=8, \ldots, 12$ (mGal)

We identify the right-hand side of the observation equation (Eq. 71) with the right-hand side of Eq. (34), i.e., we set $\boldsymbol{a}_{p}^{\mathrm{T}} \boldsymbol{\beta}=: \boldsymbol{k}_{\mathrm{rep}}\left(\boldsymbol{x}_{p}\right)^{\mathrm{T}} \boldsymbol{d}$. For the MRR of the input data, we select the Blackman scaling function defined by its Legendre coefficients

$\Phi_{j ; n}=: \Phi_{j ; n}^{\mathrm{Bla}}= \begin{cases}1 & \text { if } n \in\left[0, b^{j-1}\right) \\ A_{j}(n) & \text { if } n \in\left[b^{j-1}, b^{j}\right) \\ 0 & \text { else }\end{cases}$

wherein

$A_{j}(n)=\frac{21}{50}-\frac{1}{2} \cos \left(\frac{2 \pi n_{j}}{b_{j}}\right)+\frac{2}{25} \cos \left(\frac{4 \pi n_{j}}{b_{j}}\right)$

with $n_{j}=n+\left\lfloor b^{j}\right\rfloor-2 \cdot\left\lfloor b^{j-1}\right\rfloor, b_{j}=2 \cdot\left(b^{j}-b^{j-1}\right)$ and $b \in$ $\mathbb{R}^{+}$. Recall that in Eq. (63) the Blackman scaling function was already presented for $b=2$. Due to Eq. (52) each Blackman wavelet function $\psi_{j}^{\text {Bla }}\left(\boldsymbol{x}, \boldsymbol{x}_{k}\right)$ is related to a specific frequency band $B_{j}:=\left\{n \mid b^{j-1} \leq n<b^{j+1}\right\}$ and therefore strictly band-limited. With $b=1.55$ and $J=11$, the level-11 wavelet function covers the frequency range between spherical harmonics $n=80$ and $n=192$, i.e., we solve for signal parts up to degree $n=n_{11}=192$ and set $n^{\prime}=n_{11}$ in Eq. (30).

We relate the coefficients $d_{k}$ of the vector $\boldsymbol{d}=\boldsymbol{\beta}$ to the points $\boldsymbol{x}_{k}$ (Eq. 67) of a standard longitude-latitude grid within the computation window shown in Fig. 10. The spacing of the grid points is chosen such that the corresponding global point system $S_{N_{11}}$ is admissible according to Eq. (16).

Since we expect less variability in the residual geopotential for oceanic regions than for the continents, we subdivide the vector $\boldsymbol{\beta}$ into a sub-vector $\boldsymbol{\beta}_{1}$ collecting the coefficients $d_{k}$ related to the grid points $\boldsymbol{x}_{k}$ of the oceanic area and a second sub-vector $\boldsymbol{\beta}_{2}$ for the corresponding coefficients over land; note that as an option, the coastal area might be considered by a third subvector. We want to emphasize explicitly that splitting 


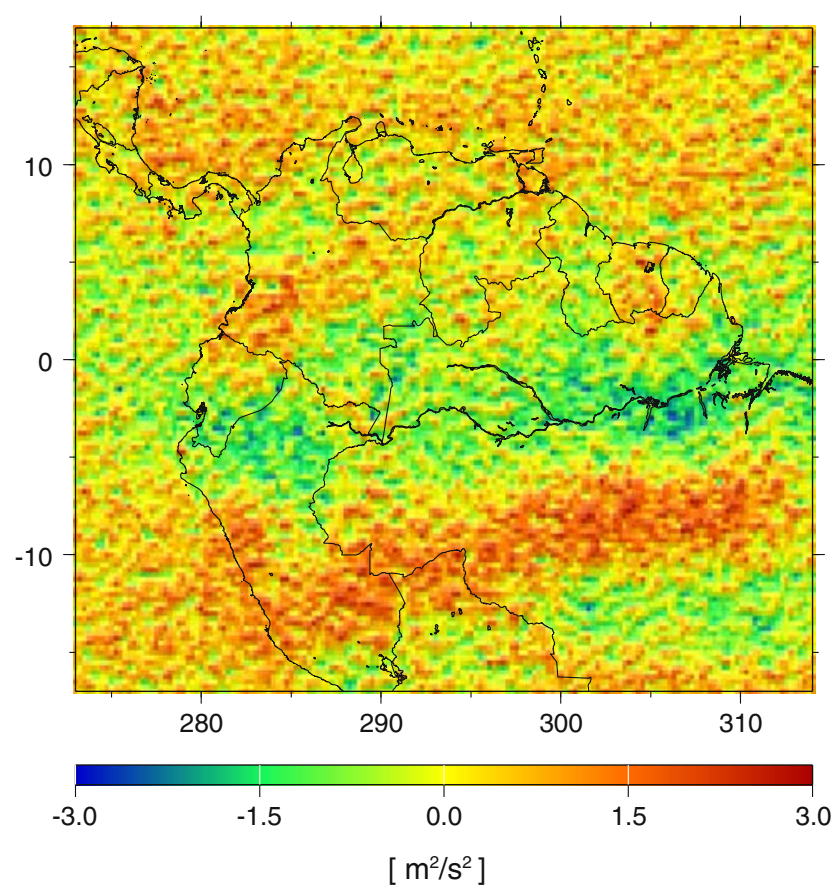

Fig. 10 Satellite input data set: CHAMP in situ potential over the northern part of South America; EGM96 up to degree and order $n=120$ is subtracted. In order to reduce boundary effects, the real data window is a little bit larger than the computation window shown here. The data was computed and kindly provided by J. van Loon, Technical University of Delft

the prior information into different spatial subregions means a space-dependent regularization.

We introduce the prior information $\boldsymbol{\mu}_{\beta_{1}}=\mathbf{0}$ and $\boldsymbol{\mu}_{\beta_{2}}=\mathbf{0}$ for the expectation vectors as well as $\boldsymbol{P}_{\beta_{1}}=\boldsymbol{I}$ and $\boldsymbol{P}_{\beta_{2}}=\boldsymbol{I}$ for the inverse covariance matrices. We use a fast Monte-Carlo implementation of the iterative maximum likelihood variance component estimation (Koch and Kusche 2001) and obtain the estimators $\widehat{\boldsymbol{\beta}}=\widehat{\boldsymbol{d}}, \widehat{\sigma}_{y}^{2}$, $\widehat{\sigma}_{\beta_{1}}^{2}$ and $\widehat{\sigma}_{\beta_{2}}^{2}$ following Eqs. (76) to (78). The estimator $\widehat{\sigma}_{\beta_{2}}^{2}$ is about twenty times larger than the estimated variance component $\widehat{\sigma}_{\beta_{1}}^{2}$, i.e., as expected the linear model (Eq. 73) for the prior information causes a much stronger regularization for the oceanic regions than for the continent. Furthermore, from Eq. (79), we obtain an estimation of the covariance matrix $D(\widehat{\boldsymbol{d}})$.

As mentioned before, the estimated coefficient vector $\widehat{\boldsymbol{d}}=\widehat{\boldsymbol{d}}_{11}$ is the input quantity of the MRR shown in Fig. 1 . The estimated detail signals $\widehat{G}_{j}(\boldsymbol{x})$ up to level $j=11$ (not shown here) are computed according to Eq. (56) and transferred into the detail signals $\widehat{\zeta}_{j}(\boldsymbol{x})=: \widehat{\zeta}_{j}^{\text {cha }}(\boldsymbol{x})$ of height anomalies following Molodensky's theory (e.g., Heiskanen and Moritz 1967). The corresponding covariance matrices are calculable as mentioned in the context of Eq. (79).

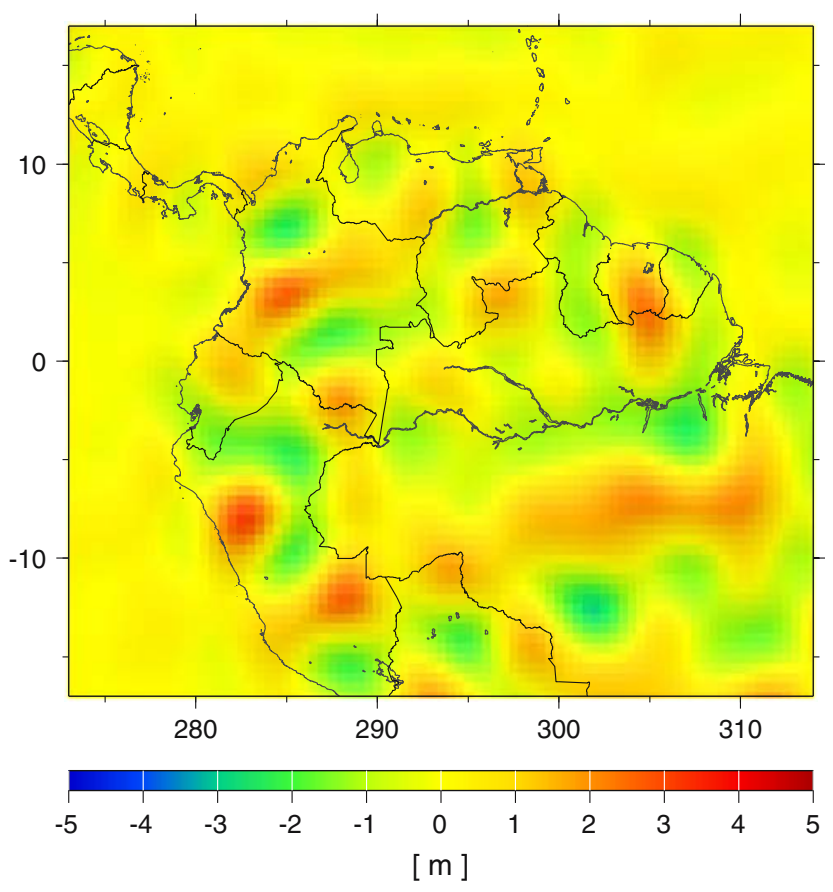

Fig. 11 Estimated residual height anomalies $\delta \widehat{\zeta}^{\text {cha }}(\boldsymbol{x})$ from the CHAMP Blackman wavelet model: residual to EGM96 to degree and order $n=120$

Figure 11 shows the estimated residual height anomalies

$\delta \widehat{\zeta}^{\mathrm{cha}}(\boldsymbol{x})=\sum_{j=2}^{11} \widehat{\zeta}_{j}^{\text {cha }}(\boldsymbol{x})$

By adding the corresponding height anomalies $\bar{\zeta}(\boldsymbol{x})$ from EGM96 up to degree $n=120$ we finally obtain the estimated height anomalies $\widehat{\zeta}^{\text {cha }}(\boldsymbol{x})=\bar{\zeta}(\boldsymbol{x})+\delta \widehat{\zeta}^{\text {cha }}(\boldsymbol{x})$.

In order to check the quality of the wavelet representation, we compare the CHAMP Blackman wavelet model (Eq. 82) with the corresponding values of the GRACE-only EIGEN-GRACE02S global spherical harmonic model from GFZ (Reigber et al. 2005). As can be seen from Figs. 11 and 12, there is a very good agreement between the two models; the RMS value of the differences amounts $0.5 \mathrm{~m}$, the correlation is approximately 0.72 . We believe the main reason for these promising results is the space-dependent regularization mentioned before instead of the frequencydependent regularization usually performed within the spherical harmonic approach.

A similar study of the same input data set was performed by Schmidt et al. (2005b). In contrast to our investigations here, they used numerical integration techniques and did not apply a regularization procedure. They derived a so-called multi-level wavelet 


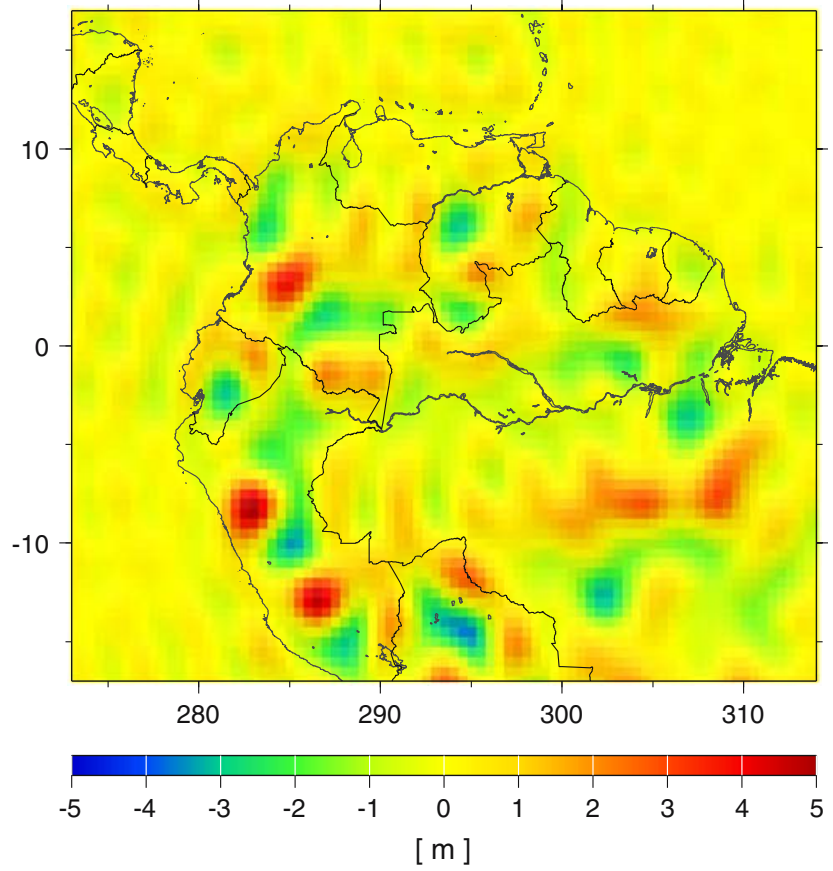

Fig. 12 Residual height anomalies computed from GFZ's GRACE-only EIGEN-GRACE02S gravity field model (EGM96 up to degree $n=120$ is subtracted)

representation of geoid undulations, i.e. they introduced different "highest" resolution levels for the oceans and the continent, respectively.

\subsection{Combined model from CHAMP and surface data}

In order to establish a high-resolution gravity model, we have to combine satellite data with surface data, since different measurement types generally cover different parts of the frequency spectrum (e.g., Kern et al. 2003). Satellite data provide the low- and medium-frequency information of the geopotential, whereas local or regional surface data cover the medium- and remaining high-frequency parts.

As surface data, we analyze a high-resolution data set containing $2^{\prime} \times 2^{\prime}$ mean Faye gravity anomalies referred to ground level (Fig. 13). This data set has been derived from terrestrial and aerial gravity measurements (Sánchez 2003) and complemented by altimetry gravity anomalies of Sandwell and Smith (1997) [gravity data $\left(2^{\prime} \times 2^{\prime}\right.$ grid $)$, version 10.2] in marine areas. Again, EGM96 complete to degree $n=120$ is removed as the reference field (Fig. 13). Recall that this data set was already used in Sect. 3.1 for studying the different scaling and wavelet functions introduced at the end of Sect. 2.4.

By means of FFT-based numerical integration techniques, already mentioned in Sect. 3.1, the decomposition into detail signals $\zeta_{j}^{\text {sur }}(\boldsymbol{x})$ of height anomalies for

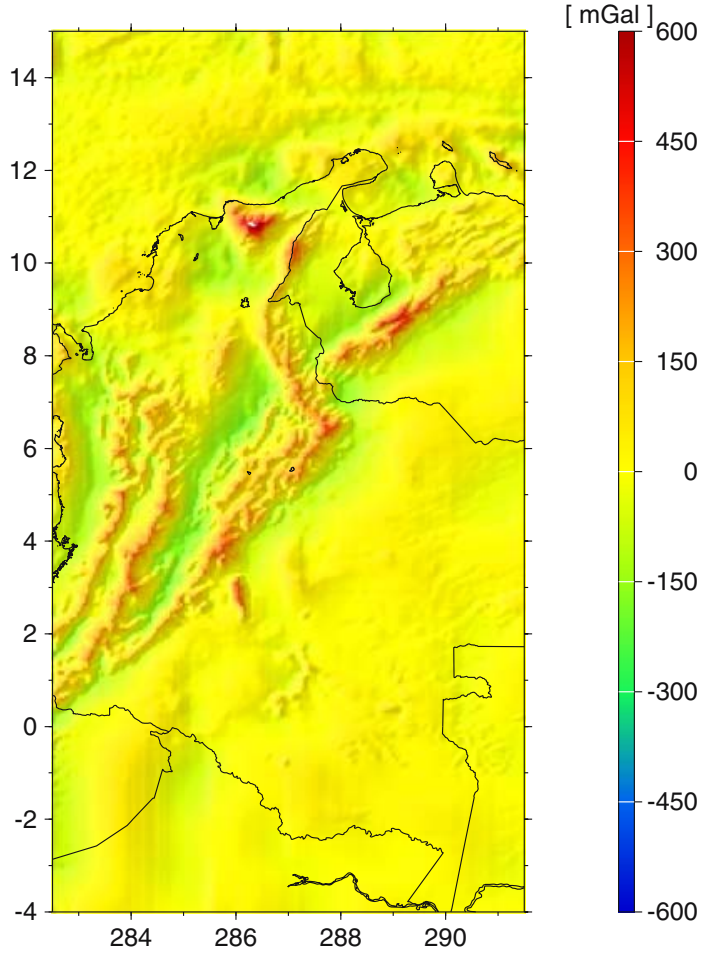

Fig. 13 Surface input data set: $2^{\prime} \times 2^{\prime}$ mean Faye gravity anomalies over Colombia; EGM96 up to degree $n=120$ is subtracted

levels $j=9, \ldots, 18$ (not shown here) is performed again using the Blackman wavelet function with $b=1.55$. Hence, the detail signal of level $j=18$ contains signal parts up to degree $n=4,133$. As mentioned before, a level-combination strategy has to be applied in order to derive the high-resolution gravity model, generally formulated as

$\zeta(\boldsymbol{x})=\bar{\zeta}(\boldsymbol{x})+\sum_{j=j^{\prime}}^{J} w_{j}^{\text {cha }} \widehat{\zeta}_{j}^{\text {cha }}(\boldsymbol{x})+\sum_{j=j^{\prime}}^{J} w_{j}^{\text {sur }} \zeta_{j}^{\text {sur }}(\boldsymbol{x})$

for the height anomaly $\zeta(\boldsymbol{x})$.

The level weights $w_{j}^{\text {cha }}$ and $w_{j}^{\text {sur }}$ are restricted to $0 \leq$ $w_{j}^{\text {cha }} \leq 1,0 \leq w_{j}^{\text {sur }} \leq 1$ and

$w_{j}^{\text {cha }}+w_{j}^{\text {sur }}=1 \quad$ for $\quad j=j^{\prime}, \ldots, J$.

Using the degree variances $\sigma_{n}^{2}(F)$, introduced in the context of Eq. (8), and the corresponding error degree variances $\epsilon_{n}^{2}(F)$, the Wiener filter curve values $p_{n}(F)$ of a signal $F$ are defined as $p_{n}(F)=\sigma_{n}^{2}(F) /\left(\sigma_{n}^{2}(F)+\epsilon_{n}^{2}(F)\right)$ (e.g., Wang 1993, Kern et al. 2003). Here we use the Wiener filter curve of GFZ's EIGEN-CHAMP03S model and determine the satellite level weights $w_{j}^{\text {cha }}$ for $j=9,10,11$ 


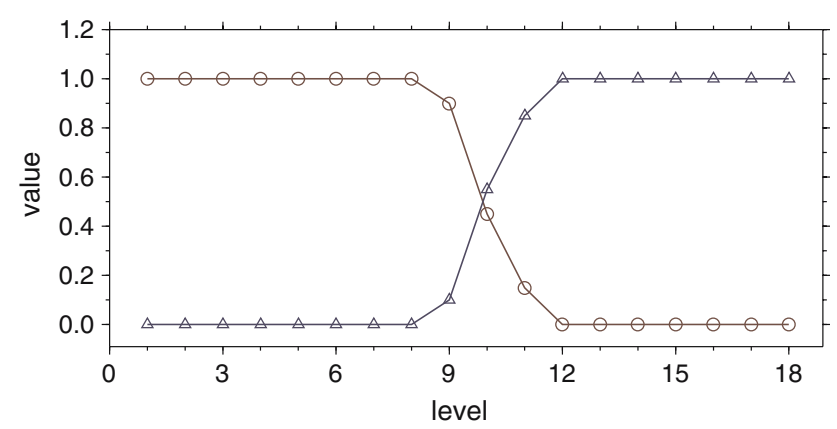

Fig. 14 Level weights $w_{j}^{\text {cha }}$ (circles) and $w_{j}^{\text {sur }}$ (triangles) of the detail signals in Eq. (83) with $j^{\prime}=1$ and $J=18$

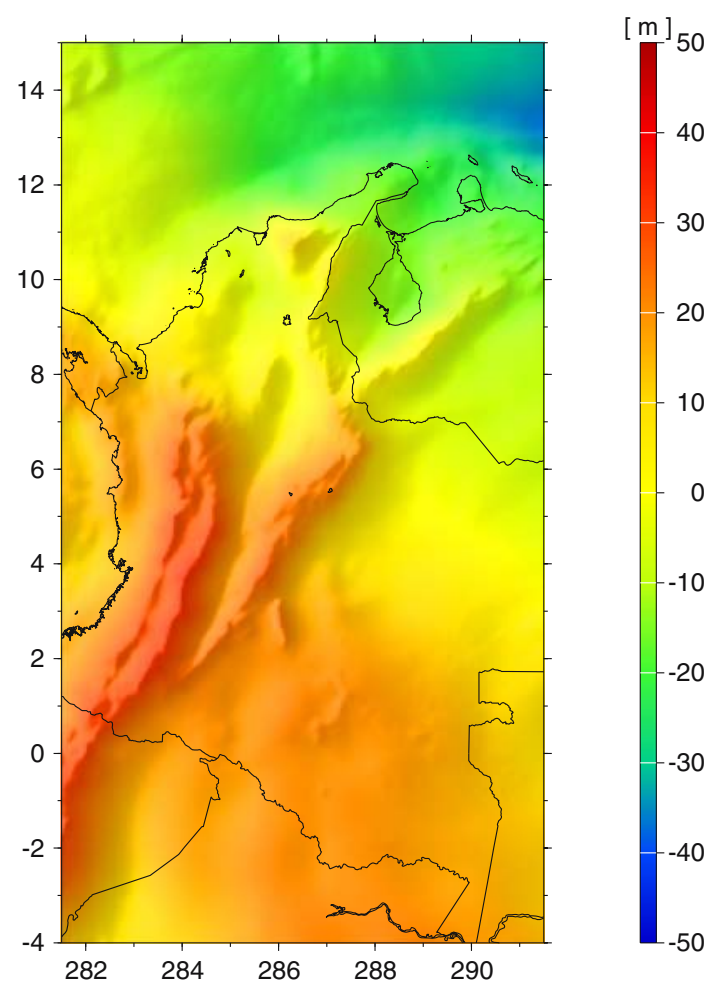

Fig. 15 High-resolution gravity field model [in terms of height anomalies (m)] of Colombia computed according to Eq. (83) with $j^{\prime}=2$ and $J=18$. The model contains signal parts up to degree $n=4,133$

according to the formula

$w_{j}^{\text {cha }}=\left(\sum_{n=0}^{n^{\prime}} p_{n}(F) \Phi_{j ; n}^{\mathrm{Bla}}\right) /\left(\sum_{n=0}^{n^{\prime}} \Phi_{j ; n}^{\mathrm{Bla}}\right) ;$

Figure 14 displays the numerical values of the level weights $w_{j}^{\text {cha }}$ and $w_{j}^{\text {sur }}$ according to Eqs. (84) and (85).

Figure 15 shows the height anomalies $\zeta(\boldsymbol{x})$ of the highresolution gravity field model of Colombia according to Eq. (83) with $j^{\prime}=2$ and $J=18$.
In contrast to the procedure described before, Schmidt et al. (2005b) computed a high-resolution gravity field model without applying a combination strategy. In other words, they chose level weights $w_{j}^{\text {cha }}=1$ and $w_{j}^{\text {sur }}=0$ for the satellite part as well as $w_{j}^{\text {cha }}=0$ and $w_{j}^{\text {sur }}=1$ for the remaining levels computed from surface data.

\subsection{GRACE-only model}

The approach presented here for deriving a geopotential model from GRACE data is based on a combined representation of spherical harmonics and harmonic splines as space-localizing base functions. It integrates a global gravity field recovery with regional gravity field refinements tailored to the local gravity field features. In a first step, the gravity field up to a moderate spherical harmonic degree is recovered; the individual gravity field characteristics in areas of rough gravity field signals are modeled subsequently by space localizing base functions in a second step.

The observation equation is based on an adjustment of the functional model that has been successfully applied to CHAMP data (Mayer-Gürr et al. 2005). It is a Fredholm integral equation of the first kind in the time domain, which represents a solution of a boundary-value problem to Newton's equation of motion for short arcs of the satellite orbit. For GRACE, the observations are precise inter-satellite measurements as ranges or rangerates. Therefore, the mathematical model can be derived by projecting the equations of relative motion to the line-of-sight connection (Mayer-Gürr et al. 2006).

The following recovery results refer to one month of range-rate measurements in August 2003. From this data set, first a global solution was calculated up to degree $n=$ 90. For the regional refinement solutions, the same mathematical model as used for the global solution has been applied except for the gravity field representation. The disturbing potential is now modeled by space-localizing base functions according to Eq. (18), with the unknown coefficients $c_{k}$ of the $N \times 1$ vector $\boldsymbol{c}=\boldsymbol{\beta}$. The base functions, as given by Eq. (19), are located on a regular grid generated by a uniform densification of an icosahedron of 20 spherical equal-area triangles. This densified grid has a mean distance between the nodal points of approximately $160 \mathrm{~km}$.

According to Eq. (19), the Legendre coefficients $B_{n}$ represent the frequency behavior of the base functions. As already explained in the context of Eq. (29), these coefficients can be related to the power spectrum of the signal that is to be modeled. Consequently, up to degree $n=90$, the error degree variances $\epsilon_{n}^{2}(F)$, as introduced 


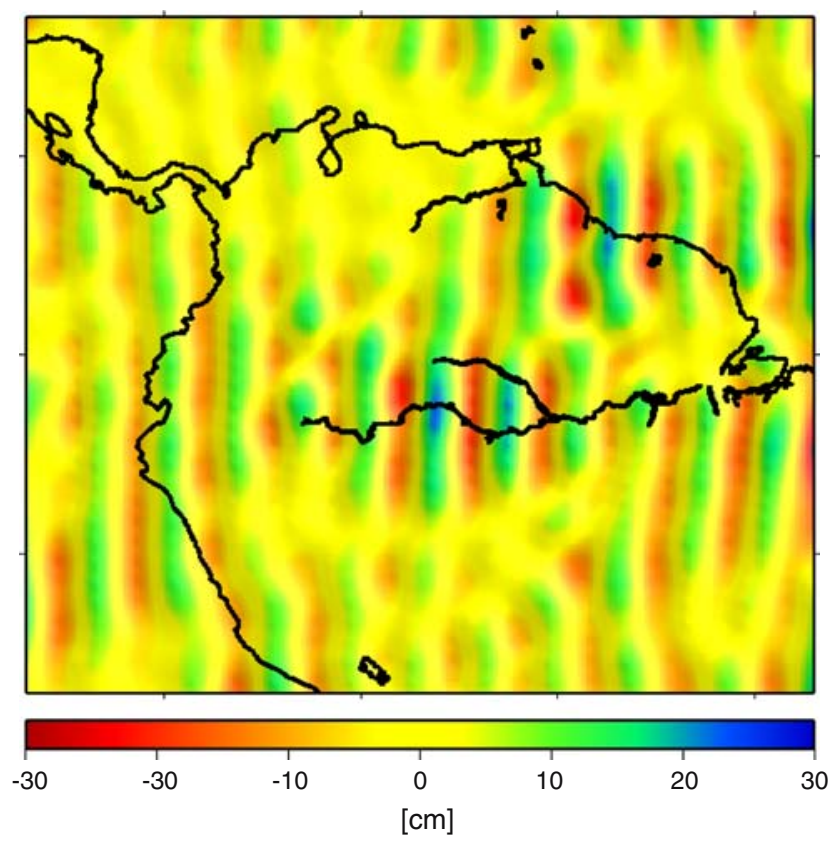

Fig. 16 Differences in geoid heights between the GRACE spline solution of August 2003 and EIGEN-CG03C; the RMS value of the differences amounts to $7.1 \mathrm{~cm}$

in the context of Eq. (85), of our global solution were used for $B_{n}$ as they represent the signal that is still in the data in addition to the global solution. Above $n=90$, the degree variances $\sigma_{n}^{2}(F)$ were calculated according to Kaula's (1966) rule of thumb. The maximum degree $n^{\prime}$ should correspond to the envisaged maximum resolution expected for the regional recovery; thus, in the following examples this maximum degree is selected as $n^{\prime}=120$, i.e.

$B_{n}= \begin{cases}\sqrt{\frac{4 \pi R^{2}}{2 n+1}} \epsilon_{n}(F) & \text { if } n \in[0,90] \\ \mathrm{GM} \sqrt{\frac{4 \pi}{2 n+1}} \frac{10^{-5}}{n^{2}} & \text { if } n \in[91,120] \\ 0 & \text { else }\end{cases}$

with GM being the product of the gravitational constant and the total mass of the Earth. To account for the illposedness of the downward continuation procedure, the method of estimating variance components is applied as already explained in Sect. 3.2 and applied in Sect. 4.1.

Figure 16 shows the differences in geoid heights of a regional refined spline solution calculated from 1 month of GRACE data compared to GFZ's combination model EIGEN-CG03 (Förste et al. 2005). The latter is based on one year of GRACE data, additional CHAMP and surface data. Thus, although considerably less data has been used for our spline solution, an RMS value of $7.1 \mathrm{~cm}$ for the differences is rather small.

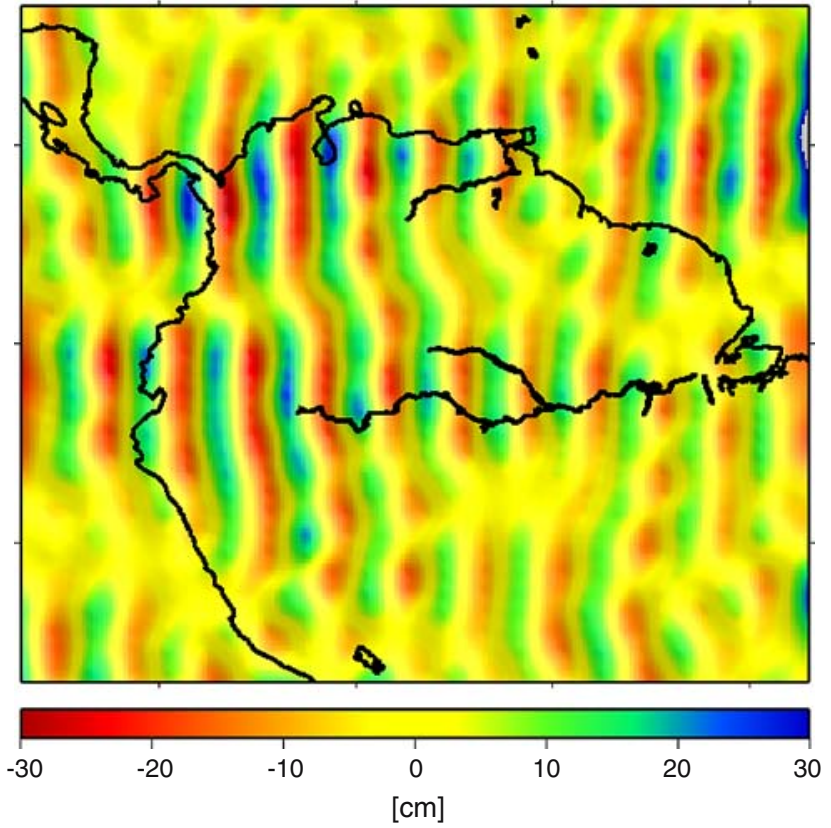

Fig. 17 Differences in geoid heights between GFZ's GRACE solution for August 2003 and EIGEN-CG03C; the RMS value of the differences amounts to $9.3 \mathrm{~cm}$

Figure 17 displays as a comparison the differences between the EIGEN-CG03C and a monthly solution calculated by GFZ for August 2003; the corresponding RMS value amounts $9.3 \mathrm{~cm}$. The stripes apparent in the residual fields may result from time-variable effects, e.g., caused by the atmosphere, that have not been modeled sufficiently. Also, due to the GRACE measurement configuration, random noise results in stripes.

Subsequently, regional refinements with an altogether global coverage can be merged by means of quadrature methods to obtain a globally refined solution without any stability problems; see Eicker et al. (2006). Using regional "zoom-ins" to calculate a global gravity field is a reasonable alternative to the direct computation of the potential coefficients since the regionally adapted recovery procedure allows for extra information to be extracted out of the given data set. In particular, it has to be pointed out that - according to Eq. (75) - the regularization parameter $\lambda$ is individually determined for each regional solution. A global regularization causes an overall filtering of the observations leading to a mean dampening of the global gravity field features. By a regionally adapted regularization, it is possible to extract more information out of the given data than would be possible with a global gravity field determination. Regions with a smooth gravity field signal for instance can be regularized more strongly without dampening the signal. In addition, the resolution of the gravity field determination can be chosen for each region individu- 
ally according to the spectral behavior of the signal. This kind of space-dependent regularization was already discussed in Sect. 4.1.

\subsection{Spatio-temporal GRACE-only model}

In Sect. 4.1, we analyzed residual geopotential values $\delta V\left(\boldsymbol{x}\left(t_{p}\right)\right)$ derived from CHAMP applying the energy balance approach. Assuming now that at time $t=t_{p}$ the two GRACE satellites are located at the positions $\boldsymbol{x}_{1}\left(t_{p}\right)$ and $\boldsymbol{x}_{2}\left(t_{p}\right)$ along their orbits, the difference $\Delta V_{1,2}\left(t_{p}\right)=$ $\delta V\left(\boldsymbol{x}_{1}\left(t_{p}\right)\right)-\delta V\left(\boldsymbol{x}_{2}\left(t_{p}\right)\right)$ means the GRACE in situ potential difference observation. Here, we define the residual geopotential values $\delta V\left(\boldsymbol{x}_{i}\left(t_{p}\right)\right)$ with $i \in\{1,2\}$ as the difference between the geopotential $V$ at position $\boldsymbol{x}_{i}\left(t_{p}\right)$ and the corresponding value $\bar{V}$ of the complete expansion of the GGM01C gravity model (Tapley et al. 2004a) chosen as the reference.

As explained by Han et al. (2006) in detail, the potential difference $\Delta V_{1,2}(t)$ can be computed by combining the inter-satellite range-rate, the position, velocity and acceleration data of the two GRACE satellites again through the energy balance approach. The observation equation for a single observation $\Delta V_{1,2}\left(t_{p}\right)=: y\left(t_{p}\right)$ is given by Eq. (71) with $\boldsymbol{a}_{p}=\boldsymbol{k}_{\mathrm{rep}}\left(\boldsymbol{x}_{1}\left(t_{p}\right)\right)-\boldsymbol{k}_{\mathrm{rep}}\left(\boldsymbol{x}_{2}\left(t_{p}\right)\right)=$ : $\boldsymbol{a}_{1,2}$ and $\boldsymbol{\beta}=\boldsymbol{d}\left(t_{p}\right)$, i.e.

$y\left(t_{p}\right)+e\left(t_{p}\right)=\boldsymbol{a}_{1,2}^{\mathrm{T}} \boldsymbol{d}\left(t_{p}\right)$.

As mass variations due to atmosphere, tidal and nontidal ocean variabilty are considered during the preprocessing steps, the observations $y\left(t_{p}\right)$ should mainly reflect the variations in continental water storage.

Our objective is now the computation of time-dependent geoid undulations $\delta N(\boldsymbol{x}, t)$. Analogous to Eq. (82), we introduce the spatio-temporal MRR

$\delta N(\boldsymbol{x}, t)=\sum_{j=j^{\prime}}^{J} N_{j}(\boldsymbol{x}, t)$

with detail signals $N_{j}(\boldsymbol{x}, t)$. By adding the corresponding values $\bar{N}(\boldsymbol{x})$ of the static reference model GGM01C, we obtain the geoid undulations $N(\boldsymbol{x}, t)=\bar{N}(\boldsymbol{x})+\delta N(\boldsymbol{x}, t)$.

Our study is again related to the northern part of South America, including the Amazon basin. The data covers the time span between February and December 2003, except June 2003. In the following, the procedure to estimate the detail signals $N_{j}(\boldsymbol{x}, t)$ from the GRACE data is described briefly; for more details see Schmidt et al. (2006).

First, we choose again the Blackman scaling function defined in Eqs. (80) and (81) with $b=2.3$ and highest
Table 1 Level-dependent observation period, total number of observations within the corresponding time-span and highest degree value $n$ related to the level $-j$ Blackman scaling function with base $b=2.3$ according to Eqs. (80) and (81)

\begin{tabular}{llll}
\hline $\begin{array}{l}\text { Level } \\
j\end{array}$ & $\begin{array}{l}\text { Observation } \\
\text { period }\end{array}$ & $\begin{array}{l}\text { Number of } \\
\text { observations }\end{array}$ & $\begin{array}{l}\text { Highest } \\
\text { degree }\end{array}$ \\
\hline 2 & 10 days & $\approx 5,000$ & 12 \\
3 & 1 month & $\approx 15,000$ & 27 \\
4 & 3 months & $\approx 25,000$ & 64 \\
\hline
\end{tabular}

resolution level $J=4$, i.e. we solve for signal parts until degree $n=64$. Hence, in Eq. (87), we have to choose the reproducing kernel (Eq. 30) with $n^{\prime} \geq 64$. In contrast to the procedure described in Sect. 4.1, we want to estimate the detail signals $N_{j}(\boldsymbol{x}, t)$ of Eq. (88) for levels $j=2,3,4$ from different data sets.

The idea stems from the fact that the determination of finer structures of the gravity field needs a denser distribution of satellite tracks than the computation of coarser structures. Hence, the estimation of the level-4 detail signal $N_{4}(\boldsymbol{x}, t)$ should be based on a longer observation period then the level-3 detail signal $N_{3}(\boldsymbol{x}, t)$. Table 1 shows the selected information to create the different data sets for establishing the desired MRR (Eq. 88).

For each data set, the parameter estimation is performed in the same manner as described in subsection 4.1, i.e., the data sets altogether provide time-series for the estimators of the scaling coefficient vectors $\boldsymbol{d}_{2}$, $\boldsymbol{d}_{3}$ and $\boldsymbol{d}_{4}$ with a temporal resolution of 10 days, 1 month and 3 months, respectively. The estimated detail signals are computed according to Eq. (56) and transformed into the detail signals $\widehat{N}_{j}(\boldsymbol{x}, t)=: \widehat{N}_{j}^{\text {gra }}(\boldsymbol{x}, t)$ by applying Bruns's theorem (e.g., Heiskanen and Moritz, 1967).

As an example, Fig. 18 shows the estimated detail signals $\widehat{N}_{3}^{\text {gra }}(\boldsymbol{x}, t)$ computed from ten data sets each covering an observation period of one month according to Table 1. Similar results can be obtained for the remaining levels $j=2$ and $j=4$ (not shown here).

Seasonal variations of the estimated geoid undulations with respect to the GGM01C reference model are clearly detectable in Fig. 18. The results agree very well with other studies on this topic; see e.g., Tapley et al. (2004b) and Han et al. (2005). We want to emphasize that in our approach, the Gaussian kernel (see the remark at the end of Sect. 2) is replaced by the Blackman kernel defined in Eqs. (80) and (81).

According to Eq. (88), the estimated variations of the gravity field can be transformed, e.g., into so-called equivalent water heights or height deformations. Schmidt et al. (2006), among others, compare these results with hydrological models and GPS time-series of height variations. 


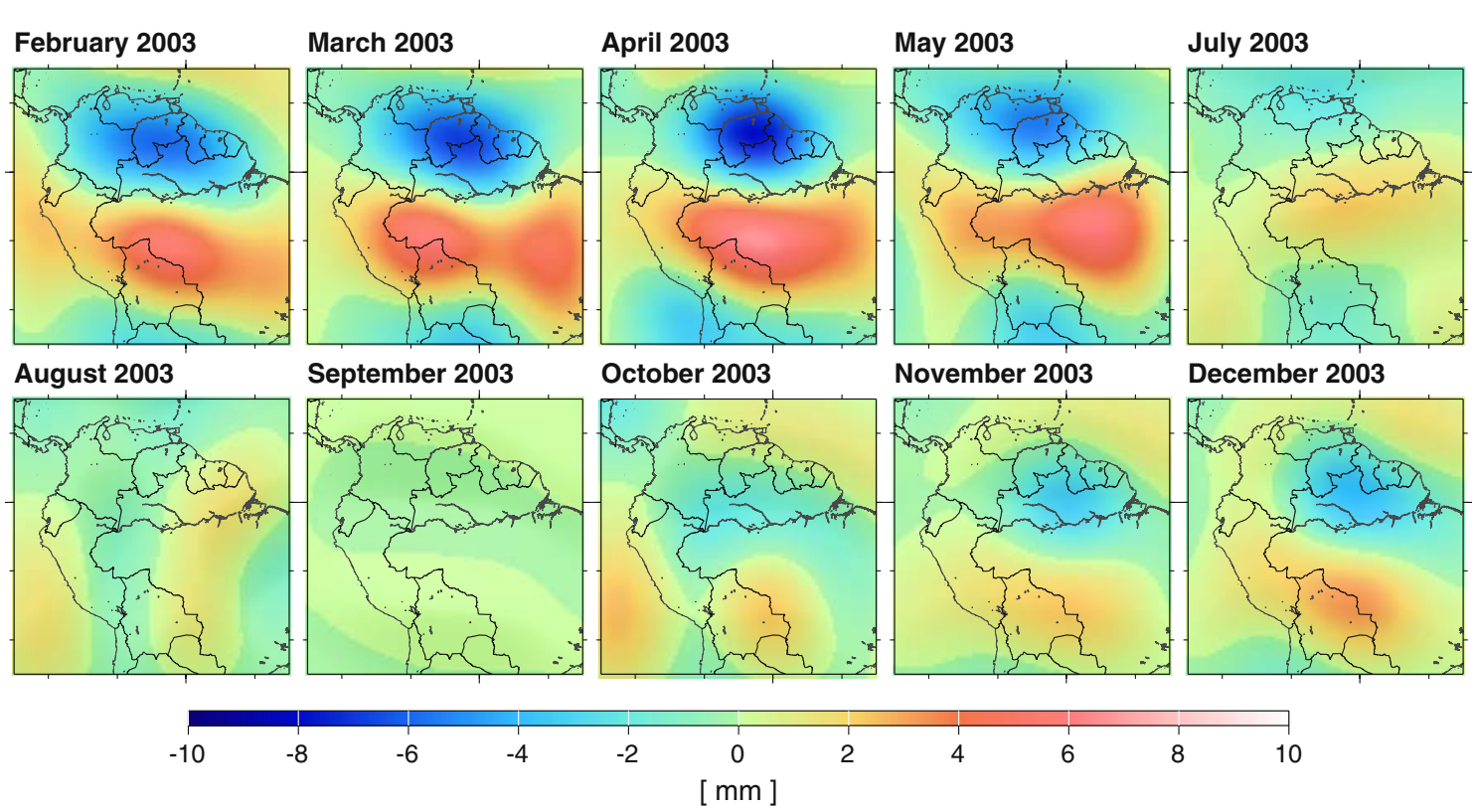

Fig. 18 Monthly solutions for detail signal $N_{3}(\boldsymbol{x}, t)$ of the residual geoid undulations $\delta N(\boldsymbol{x}, t)$ according to Eq. (88); the results are computed from GRACE data using the Blackman wavelet function with base $b=2.3$

\section{Summary and conclusions}

We have demonstrated that spline and wavelet techniques can be successfully applied to regional satellite data collected from the current CHAMP and GRACE gravity missions. We also addressed the combination of satellite and surface gravity data sets by using an appropriate weighting scheme, as well as the establishment of a spatio-temporal approach. In order to demonstrate the power of such modeling, different regional gravity representations of the northern part of South America have been derived and discussed.

One important result is the improvement of the gravity field using regional techniques in comparison with the corresponding parts of global spherical harmonic modeling. It was demonstrated that a regional wavelet CHAMP-only model fits the corresponding part of a global spherical harmonic GRACE-only model very well. The reason can be seen in the spatial regularization instead of the classical regularization in the frequencydomain. In addition, regional models with a global coverage can be merged to obtain a globally refined solution.

The multi-resolution representation allows the construction of the gravity field by detailed signals or modules. These modules are basically computable by different data sets. In spatio-temporal modeling, this procedure allows the computation of detailed signals from data sets both covering different parts of the frequency spectrum and spanning different observation intervals. Hence, this procedure means a model improvement with respect to the usual computation of time-variable gravity fields spanning fixed time intervals of, e.g., 1 month.

Acknowledgments We are grateful to GeoForschungsZentrum (GFZ) Potsdam for providing CHAMP data through their Information System and Data Center (ISDC). Thanks go also to Drazen Švehla (Technical University of Munich) for providing CHAMP kinematic orbits and to Jasper van Loon (Technical University of Delft) for processing the kinematic orbits via the energy balance approach. The authors would like to thank the anonymous reviewers for their helpful comments to improve the paper.

\section{References}

Bjerhammer A (1967) On the energy integral for satellites. Rep. of the R. Inst. of Techn. Sweden, Stockholm

Cui J (1995) Finite pointset methods on the sphere and their application in physical geodesy. PhD thesis, University of Kaiserslautern, Mathematics Department, Geomathematics Group

Driscoll JR, Healy RM (1994) Computing Fourier transforms and convolutions on the 2-sphere. Adv Appl Math 15:202250

Eicker A, Mayer-Gürr T, Ilk K-H (2006) A global CHAMP gravity field by merging of regional refinement patches. Adv Geosci (submitted) (Proceedings of the Joint CHAMP/GRACE Science Meeting)

Fengler MJ (2005) Vector spherical harmonic and vector wavelet based non-linear Galerkin schemes for solving the incompressible Navier-Stokes equation on the sphere. PhD 
thesis (submitted), University of Kaiserslautern, Mathematics Department, Geomathematics Group

Fengler MJ, Freeden W, Gutting M (2004a) The Kaiserslautern geopotential model SWITCH-03 from Orbit Pertubations of the Satellite CHAMP and its comparison to the models EGM96, UCPH2002_02_0.5, EIGEN-1S, and EIGEN-2. Geophys J Int 157:499-514

Fengler MJ, Freeden W, Kusche J (2004b) Multiscale geopotential solutions from CHAMP orbits and accelerometry. In: Reigber C, Lühr H, Schwintzer P, Wickert J (eds) Earth observation with CHAMP, results from three years in orbit. Springer, Berlin Heidelberg New York, pp 139-144

Fengler MJ, Freeden W, Gutting M (2005) The spherical Bernstein wavelet. Schriften zur Funktionalanalysis und Geomathematik, 20, University of Kaiserslautern, Mathematics Department, Geomathematics Group

Fengler MJ, Michel D, Michel V (2006a) Harmonic splinewavelets on the 3-dimensional ball and their application to the reconstruction of the Earth's density distribution from gravitational data at arbitrarily shaped orbits. ZAMM (accepted)

Fengler MJ, Freeden W, Kohlhaas A, Michel V, Peters T (2006b) Wavelet modelling of regional and temporal variations of the Earth's gravitational potential observed by GRACE. J Geod (accepted)

Förste C, Flechtner F, Schmidt R, Meyer U, Stubenvoll R, Barthelmes F, König R, Neumayer K-H, Rothacher M, Reigber Ch, Biancale R, Bruinsma S, Lemoine J-M, Raimondo JC (2005) A new high resolution global gravity field model derived from combination of GRACE and CHAMP mission and altimetry/gravimetry surface gravity data. Presented at EGU General Assembly 2005, Vienna, Austria

Freeden W (1981) On approximation by harmonic splines. manuscr geod 6:193-244

Freeden W (1999) Multiscale modelling of spaceborne geodata. Teubner, Stuttgart

Freeden W, Schreiner M (1995) New wavelet methods for approximating harmonic functions. In: Sansò F (ed) Geodetic theory today. Springer, Berlin Heidelberg New York, pp 112-121

Freeden W, Windheuser U (1996) Spherical wavelet transform and its discretization. Adv Comput Math 5:51-94

Freeden W, Michel V (2004) Multiscale potential theory (with applications to Earth's sciences). Birkhäuser Verlag, Boston

Freeden W, Gervens T, Schreiner M (1998a) Constructive approximation on the sphere (with applications to geomathematics). Clarendon Press, Oxford

Freeden W, Glockner O, Schreiner M (1998b) Spherical panel clustering and its numerical aspects. J Geod 72:586-599

Freeden W, Michel D, Michel V (2005) Local multiscale approximations of geostrophic flow: theoretical background and aspects of scientific computing. Mar Geod 28:313-329

Han SC (2003) Efficient global gravity determination from satellite-to-satellite tracking (SST). PhD thesis, Geodetic and Geoinformation Science, Department of Civil and Environmental Engineering and Geodetic Science, The Ohio State University, Columbus

Han SC, Shum CK, Jekeli C, Alsdorf D (2005) Improved estimation of terrestrial water storage changes from GRACE. Geophys Res Lett 32:L07302. DOI 10.1029/2005GL022382

Han SC, Shum CK, Jekeli C (2006) Precise estimation of in situ geopotential differences from GRACE low-low satelliteto-satellite tracking and accelerometry data. J Geophys Res 111:B4411. DOI 10.1029/2005JB003719
Heiskanen W, Moritz H (1967) Physical geodesy. Freeman, San Francisco

Holschneider M, Chambodut A, Mandea M (2003) From global to regional analysis of the magnetic field on the sphere using wavelet frames. Phys Earth Planet Int 135:107-124

Ilk KH, Löcher A (2005) The use of the energy balance relations for validation of gravity field models and orbit determination. In: Sansò F (ed) A window on the future of geodesy. Springer, Berlin Heidelberg New York, pp 494-499

Jekeli C (1981) Alternative methods to smooth the Earth's gravity field. Report 327, Department of Geodetic Science, The Ohio State University, Columbus

Jekeli C (1999) The determination of gravitational potential differences from satellite-to-satellite tracking. Cel Mech Dyn Astr 75:85-100

Kaula WM (1966) Theory of satellite geodesy. Blaisdell, Waltham

Kern M, Schwarz KP, Sneeuw N (2003) A study on the combination of satellite, airborne, and terrestrial gravity data. $\mathrm{J}$ Geod 77:217-225

Koch KR (1999) Parameter estimation and hypothesis testing in linear models. Springer, Berlin Heidelberg New York

Koch KR, Kusche J (2001) Regularization of geopotential determination from satellite data by variance components. J Geod 76:259-268

Kusche J (2002) Inverse Probleme bei der Gravitationsfeldbestimmung mittels SST- und SGG-Satellitenmissionen. German Geodetic Commission, Series C, 548, Munich

Lemoine FG, Kenyon SC, Factor JK, Trimmer RG, Pavlis NK, Chinn DS, Cox CM, Klosko SM, Luthcke SB, Torrence MH, Wang YM, Williamson RG, Pavlis EC, Rapp RH, Olson TR (1998) The development of the joint NASA GSFC and the National Imagery and Mapping Agency (NIMA) geopotential model EGM96, NASA/TP-1998-206861, National Aeronautics and Space Administration, Maryland

Li TH (1999) Multiscale representation and analysis of spherical data by spherical wavelets. SIAM J Sci Comput 21:924-953

van Loon J, Kusche J (2005) Stochastic model validation of satellite gravity data: A test with CHAMP pseudo-observations. In: Jekeli C, Bastos L, Fernandes J (eds) Gravity, geoid and space missions. Springer, Berlin Heiodelbertg New York, pp 24-29

Maier T (2005) Wavelet-Mie-representations for solenoidal vector fields with applications to ionospheric geomagnetic data. J Appl Math 65:1888-1912

Marchenko AN (1998) Parameterization of the Earth's gravity field - Point and line singularities. Lviv Astronomical and Geodetic Society, Lviv

Mautz R, Schaffrin B, Shum CK, Han SC (2004) Regional geoid undulations from CHAMP represented by locally supported basis functions. In: Reigber C, Lühr $\mathrm{H}$, Schwintzer P, Wickert J (eds) Earth observation with CHAMP, results from three years in orbit. Springer, Berlin Heidelberg New York, pp 230-236

Mayer C (2004) Wavelet modelling of the spherical inverse source problem with application to geomagnetism. Inverse problems 20:1713-1728

Mayer-Gürr T, Ilk KH, Eicker A, Feuchtinger M (2005) ITGCHAMP01: a CHAMP gravity field model from short kinematical arcs of a one-year observation period. J Geod 78:462-480

Mayer-Gürr T, Eicker A, Ilk KH (2006) Gravity field recovery from GRACE-SST data of short arcs. In: Flury J, Rummel R, Reigber C, Rothacher M, Boedecker G, Schreiber U (eds) Observation of the Earth system from Space. Springer, Berlin Heidelberg New York, pp 131-148 
Mertins A (1999) Signal analysis: wavelets, filter banks, timefrequency transforms and applications. Wiley, Chichester

Moritz H (1980) Advanced physical geodesy. Wichmann, Karlsruhe

Narcowich FJ, Ward JD (1996) Nonstationary wavelets on the m-sphere for scattered data. Appl Comput Harmon Anal 3:324-336

Panet I, Jamet O, Diament M, Chambodut A (2005) Modelling the Earth's gravity field using wavelet frames. In: Jekeli C, Bastos L, Fernandes J (eds) Gravity, geoid and space missions. Springer, Berlin Heidelberg New York, pp 48-53

Pavlis NK, Holmes SA, Kenyon SC, Schmidt D, Trimmer R (2005) A preliminary gravitational model to degree 2160. In: Jekeli C, Bastos L, Fernandes J (eds) Gravity, geoid and space missions. Springer, Berlin Heidelberg New York, pp 18-23

Reigber C, Schmidt R, Flechtner F, König R, Meyer U, Neumayer KH, Schwintzer P, Zhu SY (2005) An Earth gravity field model complete to degree and order 150 from GRACE: EIGEN-GRACE02S. J Geodyn 39(1):1-10

Sánchez L (2003) Bestimmung der Höhenreferenzfläche für Kolumbien. Diploma thesis. Institute of Planetary Geodesy, Technical University of Dresden

Sandwell DT, Smith WHF (1997) Marine gravity anomaly from Geosat and ERS-1 satellite altimetry. J Geophys Res 102(B5):10039-10054 (http://topex.ucsd.edu/www_html/ mar_grav.html)

Sansò F, Tscherning CC (2003) Fast spherical collocation: theory and examples, J Geod 77:101-112. DOI 10.1007/s00190-0020310-5

Schmidt M, Fabert O, Shum CK (2005a) Towards the estimation of a multi-resolution representattion of the gravity field based on spherical wavelets. In: Sansò F (ed) A window on the future of geodesy. Springer, Berlin Heidelberg New York, pp 362-367
Schmidt M, Kusche J, van Loon J, Shum CK, Han SC, Fabert $\mathrm{O}$ (2005b) Multi-resolution representation of regional gravity data. In: Jekeli C, Bastos L, Fernandes J (eds) Gravity, Geoid and space missions. Springer, Berlin Heidelberg New York, pp 167-172

Schmidt M, Fabert O, Shum CK (2005c) On the estimation of a multi-resolution representation of the gravity field based on spherical harmonics and wavelets. J Geodyn 39:512-526

Schmidt M, Han SC, Kusche J, Sánchez L, Shum CK (2006) Regional high-resolution spatiotemporal gravity modeling from GRACE data using spherical wavelets. Geophys Res Lett 33:L08403. DOI 10.1029/2005GL025509

Schneider F (1996) The solution of linear inverse problems in satellite geodesy by means of spherical spline approximation. J Geod 71(1):2-15

Swenson S, Wahr J (2002) Methods for inferring regional surface-mass anomalies from satellite measurements of time variable gravity. J Geophys Res 107(B9):2193. DOI 10.1029/2001JB000576

Tapley BD, Bettadpur S, Watkins M, Reigber C (2004a) The gravity recovery and climate experiment: mission overview and early results. Geophys Res Lett 31:L06619. DOI 10.1029/2003GL019285

Tapley BD, Bettadpur S, Ries J, Thompson P, Watkins M (2004b) GRACE measurements of mass variability in the Earth system. Science 305:503-505

Torge W (2001) Geodesy. de Gruyter, Berlin

Wahr J, Molenaar M, Bryan F (1998) Time variability of the earth's gravity field: hydrological and oceanic effects and their possible detection using GRACE. J Geophys Res 103(B12):30205-30229

Wang Y-M (1993) On the optimal combination of potential coefficient with terrestrial gravity data for FFT computations. manuscr geod 18:406-416 